Research Article

\title{
Meta-Analysis of miRNA Variants Associated with Susceptibility to Autoimmune Disease
}

\author{
Jun Zhang, Handan Tan, Qingfeng Cao, Guannan Su, and Peizeng Yang (D) \\ The First Affiliated Hospital of Chongqing Medical University, Chongqing Key Lab of Ophthalmology, Chongqing Eye Institute, \\ Chongqing Branch of National Clinical Research Center for Ocular Diseases, Chongqing, China \\ Correspondence should be addressed to Peizeng Yang; peizengycmu@126.com
}

Received 23 March 2021; Accepted 27 September 2021; Published 8 October 2021

Academic Editor: Margaritis Avgeris

Copyright (c) 2021 Jun Zhang et al. This is an open access article distributed under the Creative Commons Attribution License, which permits unrestricted use, distribution, and reproduction in any medium, provided the original work is properly cited.

\begin{abstract}
Purpose. Various studies have shown an association between miRNA polymorphisms and susceptibility to autoimmune disease (AD); however, the results are inconclusive. To evaluate whether miRNA polymorphisms account for a significant risk of AD, a total of 87 articles, including 39431 patients and 56708 controls, were identified to estimate their association with 12 AD subtypes. Methods. Several electronic databases were searched to analyze population-based studies on the relationship between miRNA variants and $\mathrm{AD}$ risk. Fixed effects or random effect models were used in the meta-analysis for the risk assessment. Results. In our meta-analysis, miR-146a rs2910164/rs57095329 conferred a marginally elevated risk for AD (allele model, $\mathrm{OR}=1.08,95 \% \mathrm{CI}$ : 1.01-1.15, $P=0.019$; allele model, $\mathrm{OR}=1.09$, 95 CI: $1.05-1.15, P<0.001$, respectively). Furthermore, miR-196a2 rs11614913 was also associated with AD risk (allele model, OR $=0.92,95 \%$ CI: $0.88-0.97, P=0.001$ ) as well as miR-499 rs3746444 (allele model, OR=1.16, 95\% CI: 1.03-1.29, $P=0.011$ ). In addition, associations were observed between miR-149 rs2292832/miR-27a rs895819 and AD susceptibility in the overall population (allele model, OR $=1.15,95 \%$ CI: 1.06-1.24, $P<0.001$; allele model, $\mathrm{OR}=1.11,95 \% \mathrm{CI}: 1.01-1.22, P=0.043$, respectively). Conclusions. Evidence from our systematic review suggests that miR-146a, miR-196a2, miR-499, miR-149, and miR-27a polymorphisms are associated with susceptibility to $\mathrm{AD}$.
\end{abstract}

\section{Introduction}

Autoimmune diseases $(\mathrm{AD})$ are a spectrum of disorders initiated by impaired self-tolerance of the immune system and may lead to tissue destruction, chronic inflammation, and morbidity [1]. More than 80 types of $\mathrm{AD}$ have been confirmed and affect approximately $5-10 \%$ of the total population [2]. Of note, women constitute approximately $78 \%$ of those affected individuals and bear a disproportionate burden of the high morbidity [3]. Like many other complex diseases, $\mathrm{AD}$ are believed to arise from multiple environmental and genetic factors, both of which may be shared across many AD [4]. Common nonsteroidal antiinflammatory drugs, immunosuppressants, and antitumor necrosis factor-alpha agent could be utilized for the treatment of various AD. While some patients did not respond to these treatments, suggesting other factors such as genetic background may account for this heterogeneity [5]. Associa- tion and linkage analysis in different populations have demonstrated that hereditary variation of $\mathrm{AD}$ has intrinsic commonality. Most of the mutations are typically located in either coding gene regions with an influence on protein function or noncoding gene regions, potentially affecting a targeted gene transcript. Additionally, recent studies suggested that functional variants occurring in microRNA sequences were associated with susceptibility to $\mathrm{AD}$ [6-15]. These findings indicate that variants in common miRNAs could be genetic markers of AD such as multiple sclerosis, rheumatoid arthritis, and ankylosing spondylitis, which highlighted a new paradigm for genetic susceptibility.

MicroRNAs (miRNAs) are endogenously generated single-stranded noncoding RNA molecules of about 22 nucleotides that play a pivotal role in regulating transcription and posttranscription of specific gene expression, including genes of the mammalian immune system [16]. Genetic ablation of the miRNA machinery, as well as various 
single nucleotide polymorphisms (SNPs) resulting in the loss or dysregulation of miRNAs (miRNA-SNPs), may affect immune development, differentiation, and response, which could ultimately lead to loss of immune tolerance and autoimmunity [16, 17]. Furthermore, functional miRNA-SNPs may act as potential biomarkers to predict clinical outcome or susceptibility of $\mathrm{AD}[18,19]$. Growing evidence indicated that studies on miRNA function have been moved to molecular mechanism level. miRNA could inhibit translation at the initiation step, likely involving the $\mathrm{m} 7 \mathrm{G}$ cap structure or implicating the cap-binding protein eukaryotic initiation factor [20, 21]. Additionally, miRNA also inhibits actively translating polyribosomes or ribosome drop during ongoing translation $[22,23]$.

A robust quantification of the correlation regarding the miRNA-SNPs in patients with AD risk may increase our understanding whether genetic mutations in miRNA sequence are associated with immune-related diseases. Whether shared genetic variations may have similar effects on the risk of different $\mathrm{AD}$ or whether these effects are specific for certain $\mathrm{AD}$ has not yet been investigated at the genotype level. Although several meta-analyses have already addressed the impact of miRNA polymorphisms on $\mathrm{AD}$ risk [24-27], results have been controversial and often lacked sufficient statistical power. Apparently, a review of more recent studies is required to enhance the existing knowledge and clarify the observed inconsistencies. Using novel metaanalysis techniques, we readdressed this subject to evaluate the association between common miRNA-SNPs with susceptibility to $\mathrm{AD}$.

\section{Materials and Methods}

2.1. Search Strategy. Studies reporting miRNA disease associations were retrieved from various databases including PubMed, Embase, Web of Science, Google Scholar, and the Chinese National Knowledge Infrastructure (CNKI, http://www.cnki .net/) registry, using the following keywords: "polymorphism," "SNP," "variant," "genotype," "autoimmune," "immunerelated," "miRNA," "microRNA," and "microRNAs." Each database was screened from the inception date to December 10,2020 . There were no restrictions as to language, ethnicity, or publication year. Additionally, the citations of retrieved articles were also manually scrutinized for original data sources. The Preferred Reporting Items for Systematic Reviews and Meta-Analyses (PRISMA) checklist including the page number for each item can be available in Supplementary Materials (available here).

2.2. Selection Criteria. Full length articles were reviewed for relevant keywords in the title, abstract, or keyword list. Publications were checked in the first round using the following inclusion criteria: (1) assessment of all miRNA genetic polymorphism association studies, (2) independent case-control study, and (3) having enough data to enable calculating odds ratios (OR) with $95 \%$ confidence intervals (95\% CI). Articles in the category of reviews, meta-analysis, organizational guidelines, editorial letters, expert opinions, conference abstracts, case reports, and those with insufficient raw data (after contacting the corresponding author) were excluded to avoid duplication and erroneous weighting towards more frequently cited publications. Full text screening of all studies conforming to the above criteria was performed independently by two reviewers (J.Z. and H.T.). Any discrepancy was addressed with a third reviewer (Q.C.) to reach a consensus.

2.3. Data Extraction and Quality Assessment. A data extraction sheet based on a predetermined standard, including the first author, publication year, type of disease, country, ethnicity, genotyping methods, characteristics of cases and controls, Hardy-Weinberg equilibrium (HWE) in controls, and the modified Newcastle-Ottawa Quality Assessment Scale (NOS), was compiled for each selected study. A study with a NOS score of seven or more points was considered high quality, and those with nine points were ranked the most senior. A kappa value was calculated to compare the data retrieved by the two reviewers (J.Z. and H.T.) [28]. The Grading of Recommendations, Assessment, Development, and Evaluation (GRADE) system was adopted to evaluate the quality of evidence of the included studies [29]. After going through these quality checks, a final list of studies was produced.

2.4. Data Synthesis and Meta-Analysis. In our study, the term meta-analysis included our analysis of all miRNA SNPs in the combined $\mathrm{AD}$ groups. In the subgroup analysis of specific diseases, sometimes only one study could be found and as such would not meet the definition of a meta-analysis. MantelHaenszel OR with 95\% CI was computed from the initial raw data; heterogeneity was measured by exploring the study-specific Cochran's $Q$ value $(P<0.1$, treated as significant level across all reviews) and quantitative Higgins's $I^{2}$ statistic. When the $I^{2}$ statistic was higher than $75 \%, 50 \%$, and $25 \%$, it represented large, moderate, and small heterogeneity, respectively. Thus, either the fixed effects model $\left(I^{2}<50 \%\right.$ and $P>0.1)$ or random effects model $\left(I^{2} \geq 50 \%\right.$ and $\left.P<0.1\right)$ was utilized to measure the pooled ORs and 95\% CIs. Furthermore, $I^{2}$ offers advantages over Cochran's $Q$ statistic and $I^{2}$ is preferable to a test for heterogeneity in assessing inconsistency across studies [30]. A chi-square test was conducted in controls to evaluate the deviation from HWE. Subgroup analysis was used to test the influence of the categorical moderators. Additionally, metaregression was used to evaluate the contribution of different covariables to heterogeneity. Dummy variables were applied where features had three or more outcomes (for example, ethnicity, disease type, and genotype method). A sensitivity analysis was implemented to assess the influence of each study on the pooled effect size by taking out one study in each turn. Publication bias was tested by Begg's funnel plot and Egger's regression method. A $P$ value less than 0.05 was treated as significant in these comparisons, and all statistical analyses were achieved by STATA V.12.0 software (Stata Corp LP, College Station, Texas, USA).

\section{Results}

3.1. General Characteristics of Selected Studies and Quality Assessment. The initial search retrieved 1478 publications 
from PubMed, 1603 from Embase, 892 from the Web of Science, 976 from Google Scholar, and 59 from the CNKI. Three extra articles were obtained by scanning the references of preliminary papers [31-33]. The detailed step by step of our searching strategy is drawn as a PRISMA flowchart in Figure 1. 5011 articles were screened from the databases, and 878 duplicates were excluded. After a careful choice of papers following review of titles, abstracts, and key terms related to miRNA-SNP or AD, 3983 studies were deleted for not addressing neither miRNA-SNP nor AD, and 150 full-text articles were identified to be potentially relevant. Among them, 56 studies were eliminated since they did not contain sufficient genotype results $(n=18)$ or for not investigating an association between miRNA-SNP and AD risk $(n=17)$ or for being a review or meta-analysis $(n=13)$, expert opinion $(n=3)$, or case report $(n=5)$. Ultimately, 94 articles met all the inclusion criteria for our meta-analysis, of which 87 were eligible for quantitative analysis of risk estimates [6-15, 31-107]. Assessment of interinvestigator agreement using kappa values for the selected articles yielded values of 0.84 for PubMed, 0.88 for Embase, 0.90 for Web of Science, 0.91 for Google Scholar, and 1.0 for CNKI, suggesting a high level of agreement between our two reviewers.

In our meta-analysis, AD were classified into twelve disease subgroups, including autoimmune thyroiditis (AITD), arthritis, asthma, systemic lupus erythematosus (SLE), uveitis, inflammatory bowel disease (IBD), Immunoglobulin A (IgA) nephropathy, Kawasaki disease (KD), sclerosis, type 1 diabetes mellitus (T1DM), polymyositis, and psoriasis based on the common syndrome and disease homogeneity risk [108]. We included asthma as an $\mathrm{AD}$, because it is triggered not only by allergen exposure but also by other mechanisms, possibly autoreactive/autoimmune. This classification is further supported by the response to immunosuppressive drugs. The distribution of total patients in the overall subgroups is delineated in Figure 2, and the top five include SLE (32.6\%), arthritis (15.9\%), uveitis (15.5\%), psoriasis $(6.9 \%)$, and $\mathrm{KD}(5.7 \%)$. Furthermore, ethnic origins were categorized as Caucasian, East Asian, Hispanic, Middle East, and Oceanian. The basic characteristic of each study is shown in Table 1. Only SNPs with a minor allele frequency greater than $5 \%$ of the control population were included. The genotype frequencies of the controls in all studies, except seven articles, conformed to HWE $(P>0.05)$. Apart from two papers, the quality of the evidence generally received a score ranging from five to nine by the NOS criteria. All included studies were graded as "low" quality according to the GRADE profiler, except for one study [81] which was graded as "very low." Low gradings were due to the observational design of studies, putting them at risk of bias, imprecision, and inconsistency.

3.2. Quantitative Data Synthesis and Meta-Analysis. 87 articles describing 109 studies with 39431 patients and 56708 controls were finally included. Pooling these data, we estimated the miRNA-SNP risk for $23 \mathrm{AD}$ and accomplished a meta-analysis of $12 \mathrm{AD}$ subtypes into the case group. The following paragraphs discuss the epidemiological studies and summarize the genetic susceptibility to AD (Table 2, supplementary materials (available here)).

3.2.1. miR-146a. A total of four SNPs (rs2910164, rs57095329, rs2431697, and rs6864584) in the miR-146a gene were investigated from data retrieved from 71 studies. Meta-analysis indicated that the G allele of rs2910164 was positively associated with $\mathrm{AD}$ susceptibility in the overall population (allele model, $\mathrm{OR}=1.08,95 \% \mathrm{CI}$ : 1.01-1.15, $P=0.019$, Figure 3; dominant model, $\mathrm{OR}=1.09,95 \%$ CI: $1.01-1.20, P=0.049)$. After stratifying by disease subtype, it was associated with a decreased risk of IBD (allele model, $\mathrm{OR}=0.79,95 \% \mathrm{CI}: 0.65-0.97, P=0.027$; dominant model, $\mathrm{OR}=0.78,95 \% \mathrm{CI}: 0.65-0.92, P=0.001$; recessive model, $\mathrm{OR}=0.67,95 \% \mathrm{CI}: 0.50-0.88, P=0.005)$. On the contrary, it was correlated with increased risk of arthritis (allele model, $\mathrm{OR}=1.15,95 \%$ CI: $1.01-1.31, P=0.034$; recessive model, OR $=1.17,95 \% \mathrm{CI}: 1.01-1.36, P=0.048)$, with asthma (allele model, OR $=1.16,95 \% \mathrm{CI}: 1.03-1.31, P=0.014$; dominant model, $\mathrm{OR}=1.25,95 \% \mathrm{CI}: 1.02-1.54, P=0.029)$, with uveitis (allele model, $\mathrm{OR}=1.44,95 \% \mathrm{CI}$ : 1.14-1.81, $P=0.002$; dominant model, $\mathrm{OR}=1.26,95 \% \mathrm{CI}: 1.13-1.40, P<0.001$; recessive model, $\mathrm{OR}=1.73,95 \% \mathrm{CI}: 1.21-2.47, P=0.003)$, and with psoriasis (allele model, OR $=1.15,95 \% \mathrm{CI}$ : 1.05 $1.25, P=0.001$; dominant model, $\mathrm{OR}=1.38$, 95\% CI: 1.13 $1.69, P=0.002)$. A stratified analysis by ethnicity revealed a significant increase in the risk of $\mathrm{AD}$ in the Middle East population (especially in the allele model, $\mathrm{OR}=1.66,95 \% \mathrm{CI}$ : 1.35-2.04, $P<0.001$; dominant model, $\mathrm{OR}=2.54, \quad 95 \%$ CI: $1.60-4.02, P<0.001$; recessive model, $\mathrm{OR}=2.13,95 \%$ CI: 1.54-2.95, $P<0.001)$, but a decreased risk in the Caucasian and Oceanian groups using the recessive model (OR $=0.86,95 \%$ CI: $0.75-0.99, P=0.036 ; \mathrm{OR}=0.78,95 \%$ CI: $0.61-0.99, P=0.037$, respectively). Subgroup metaanalysis by methodological quality of the studies as ranked by the NOS scale revealed no significant positive association in neither the high-quality studies nor the low-quality studies (shown in supplementary materials (available here)).

Furthermore, an elevated risk of $\mathrm{AD}$ was found in subjects with the rs57095329 $\mathrm{G}$ allele model $(\mathrm{OR}=1.09,95 \mathrm{CI}$ : 1.05-1.15, $P<0.001)$. In addition, a stratified analysis based on disease subtype showed that this variant conferred an increased risk of SLE in the allele model and a decreased risk of sclerosis in the recessive model. In the stratified analysis by ethnicity, a significant relationship was detected in the East Asian and Middle East groups (shown in supplementary materials (available here)).

Moreover, pooled results showed that the $\mathrm{C}$ allele of rs2431697 was associated with a significantly decreased risk of $\mathrm{AD}$ in the overall population (allele model, $\mathrm{OR}=0.77,95 \%$ CI: 0.71-0.84, $P<0.001$; dominant model, $\mathrm{OR}=0.74,95 \% \mathrm{CI}$ : $0.56-0.98, P=0.037$; recessive model, $\mathrm{OR}=0.76,95 \% \mathrm{CI}$ : $0.62-0.92, P=0.006)$. Based on the disease subtype, an obvious association was found in SLE among three genetic models. Our stratified analysis results revealed a significant association in the East Asian and Caucasian groups (shown in supplementary materials (available here)).

Lastly, meta-analysis suggested that the rs6864584 C allele was associated with a decreased risk of $\mathrm{AD}$ in the total 


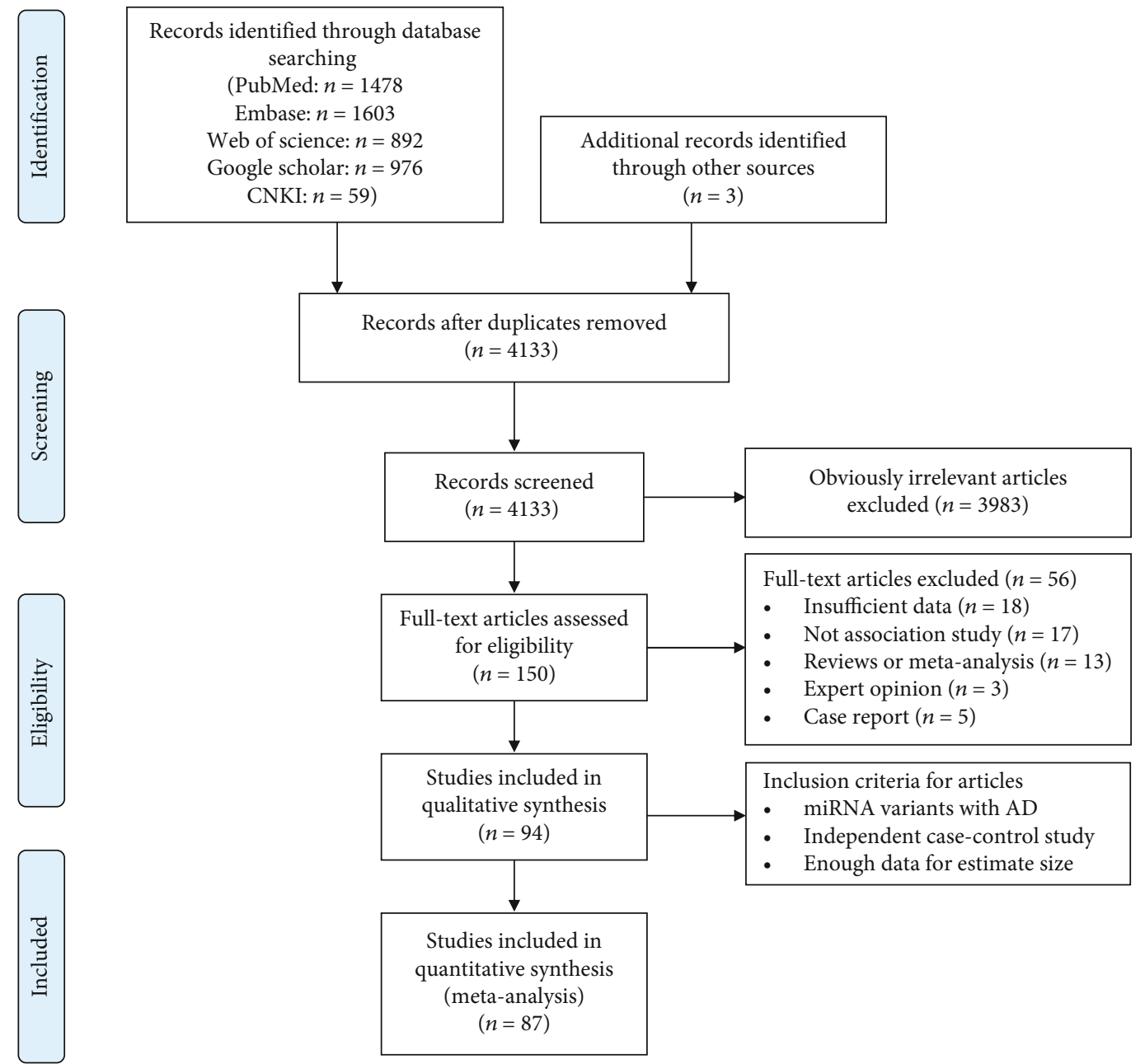

FIGURE 1: Flow diagram presenting the result of literature searching process in meta-analysis.

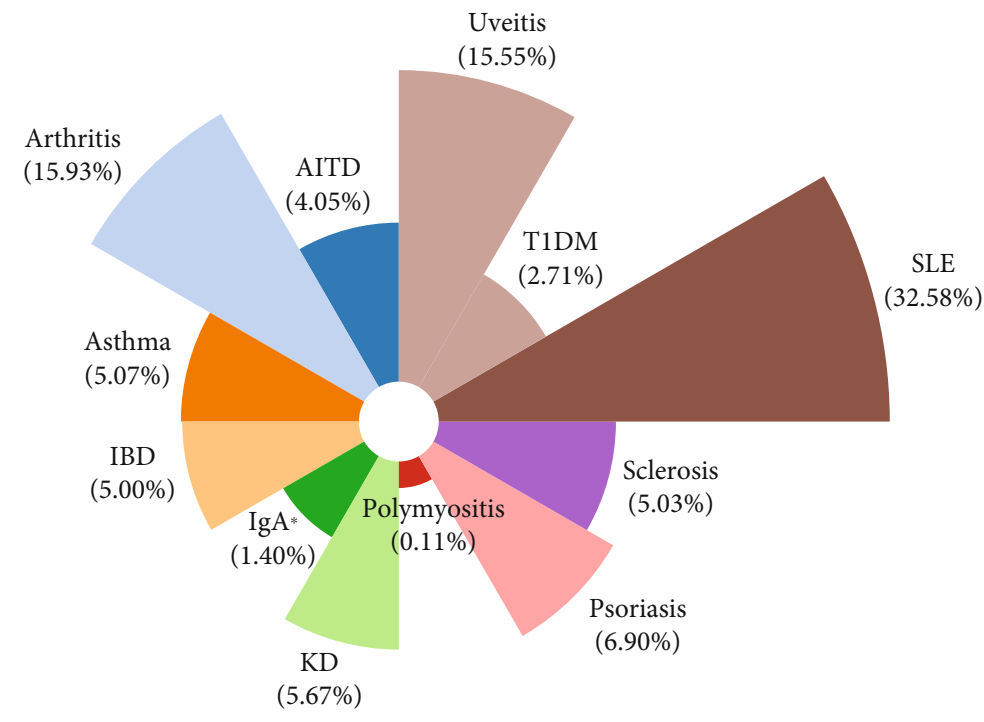

FIGURE 2: The distribution of total patients in the overall subgroups. 


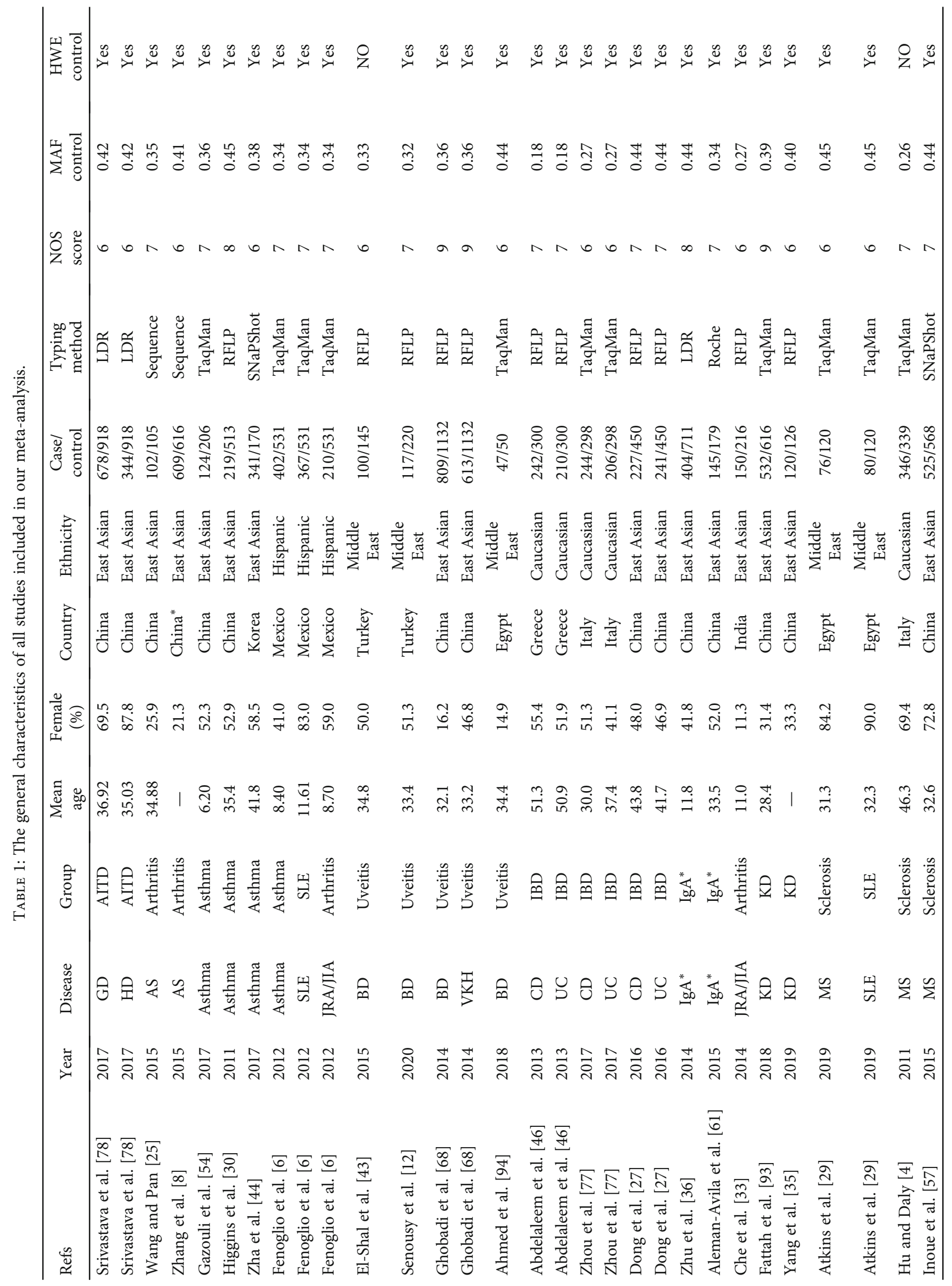




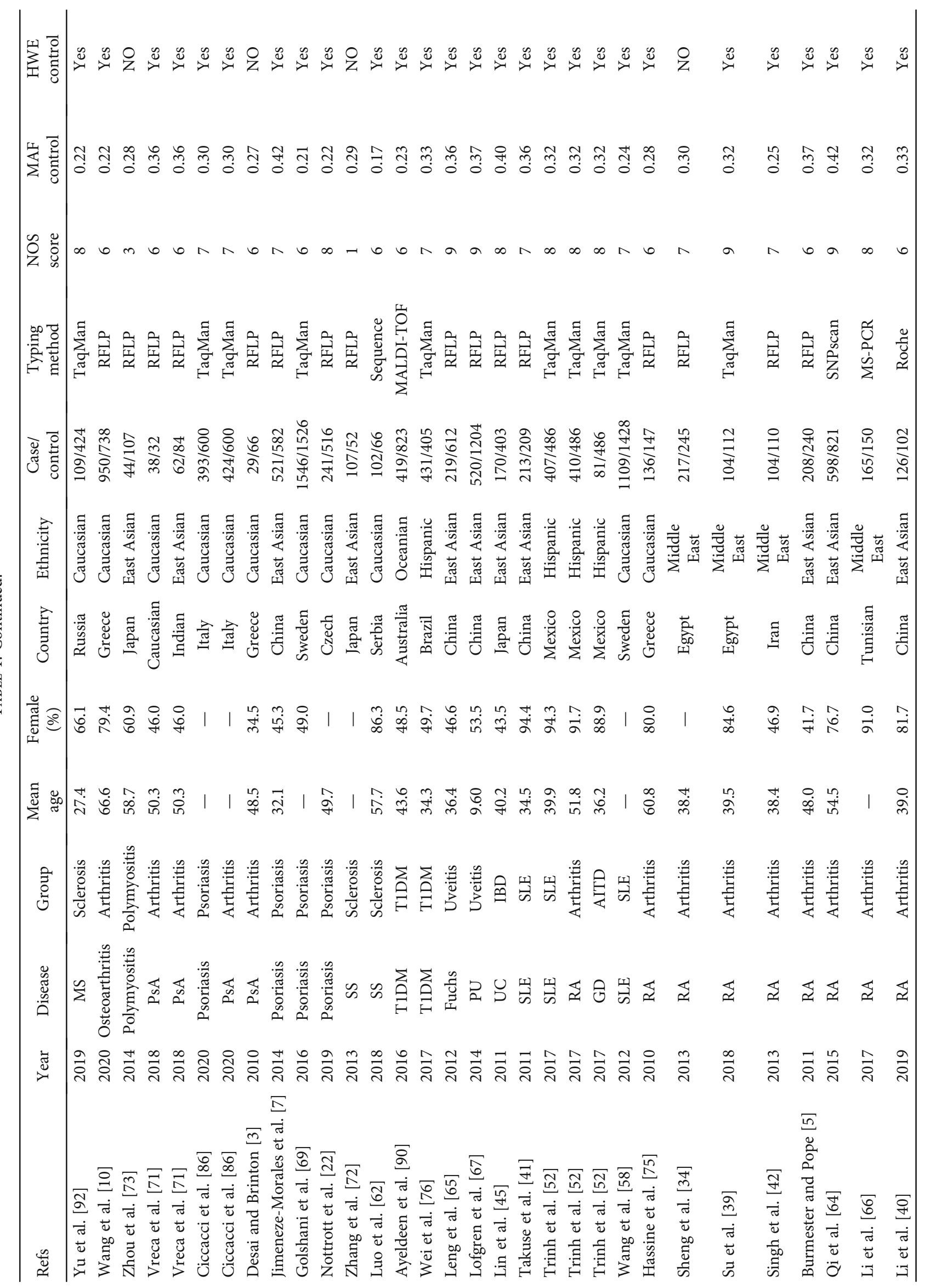




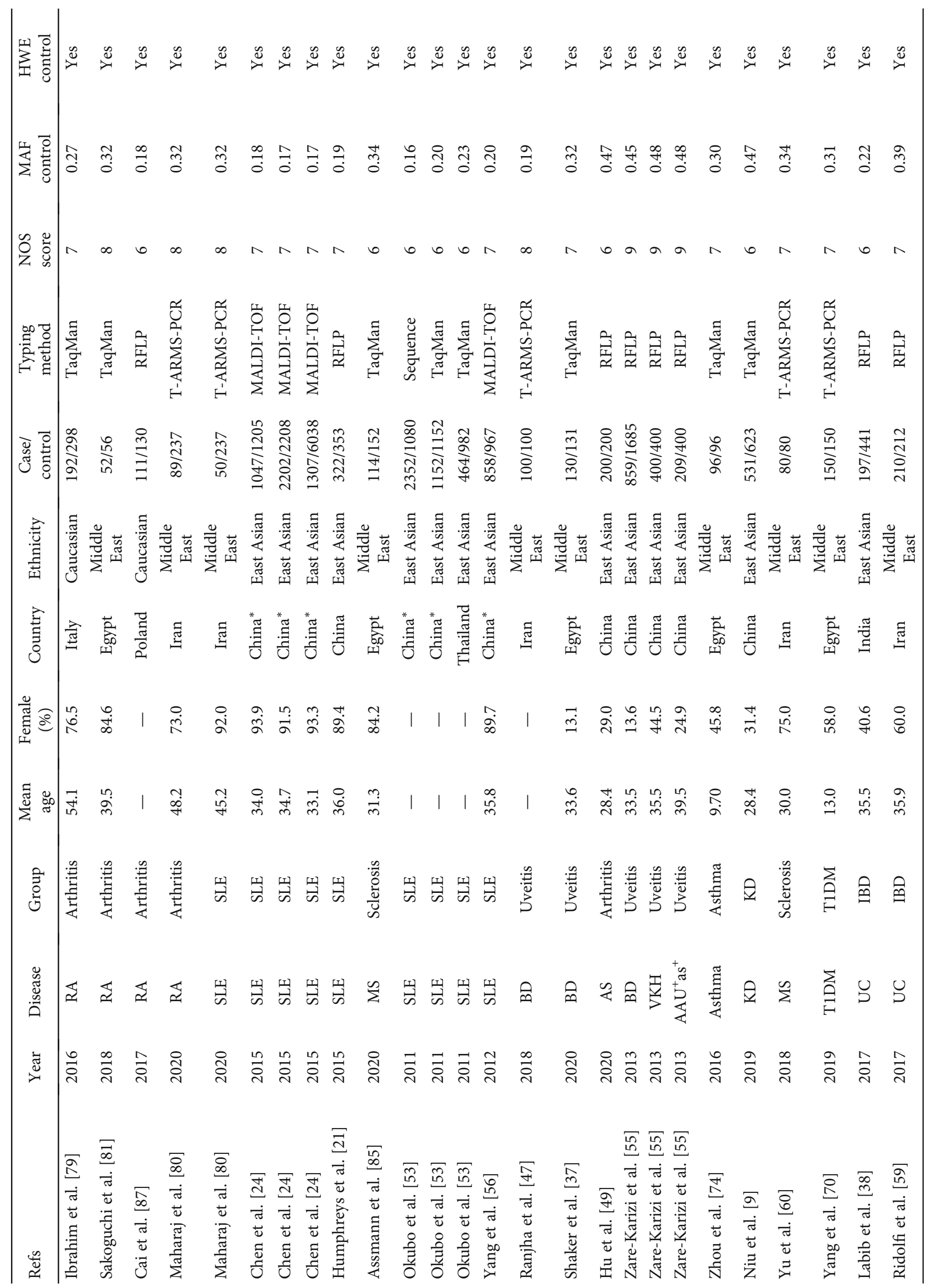




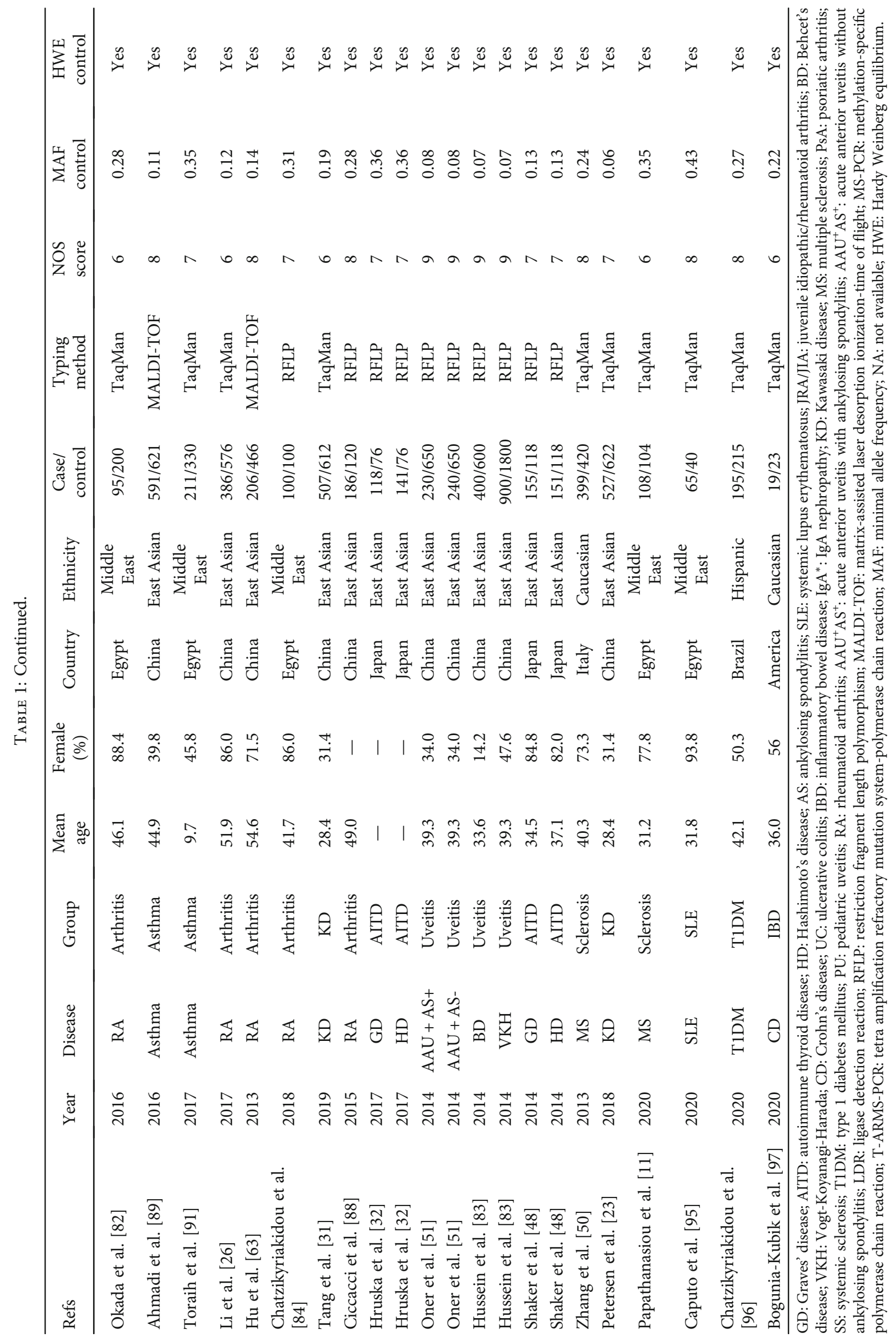




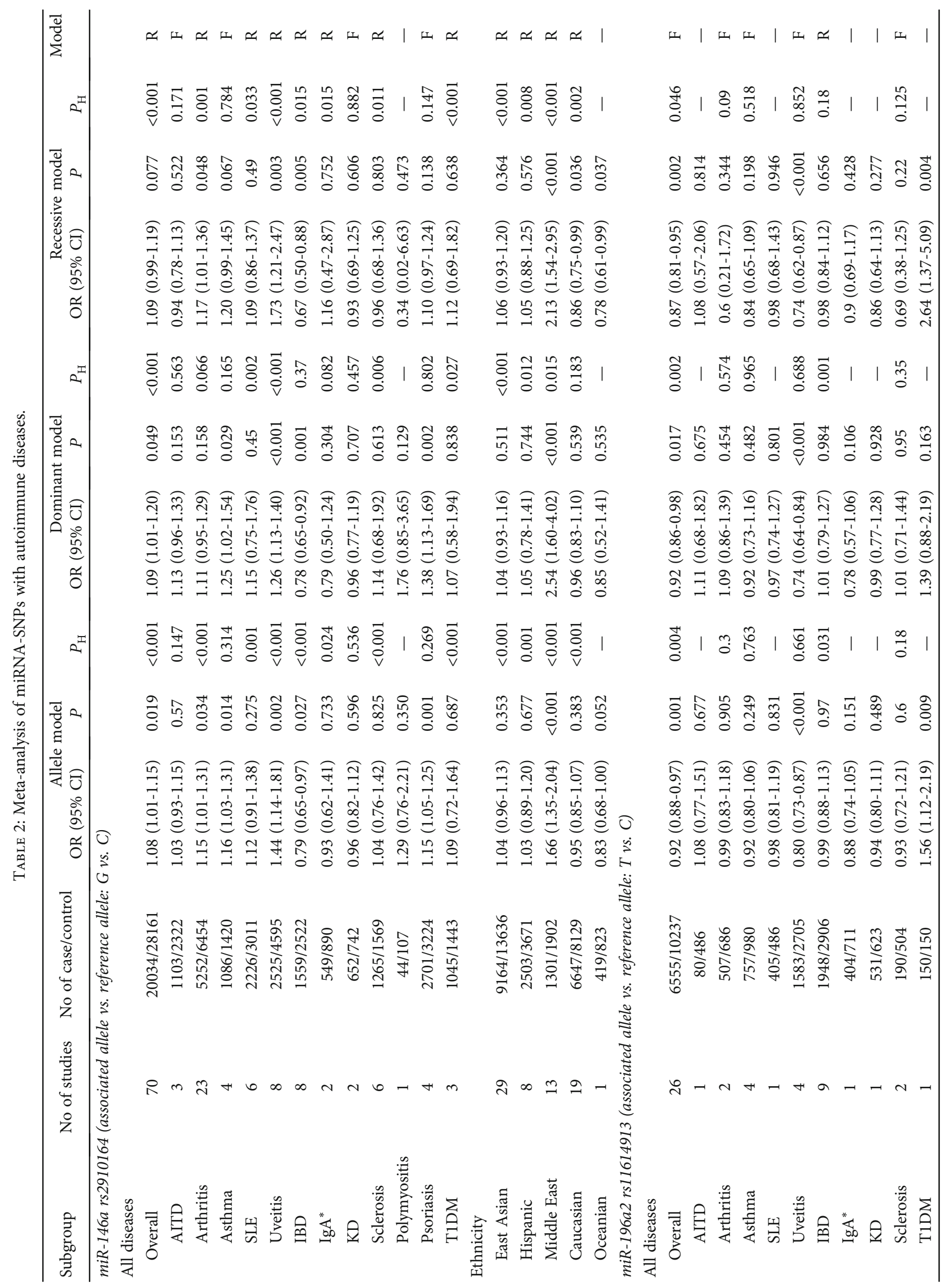




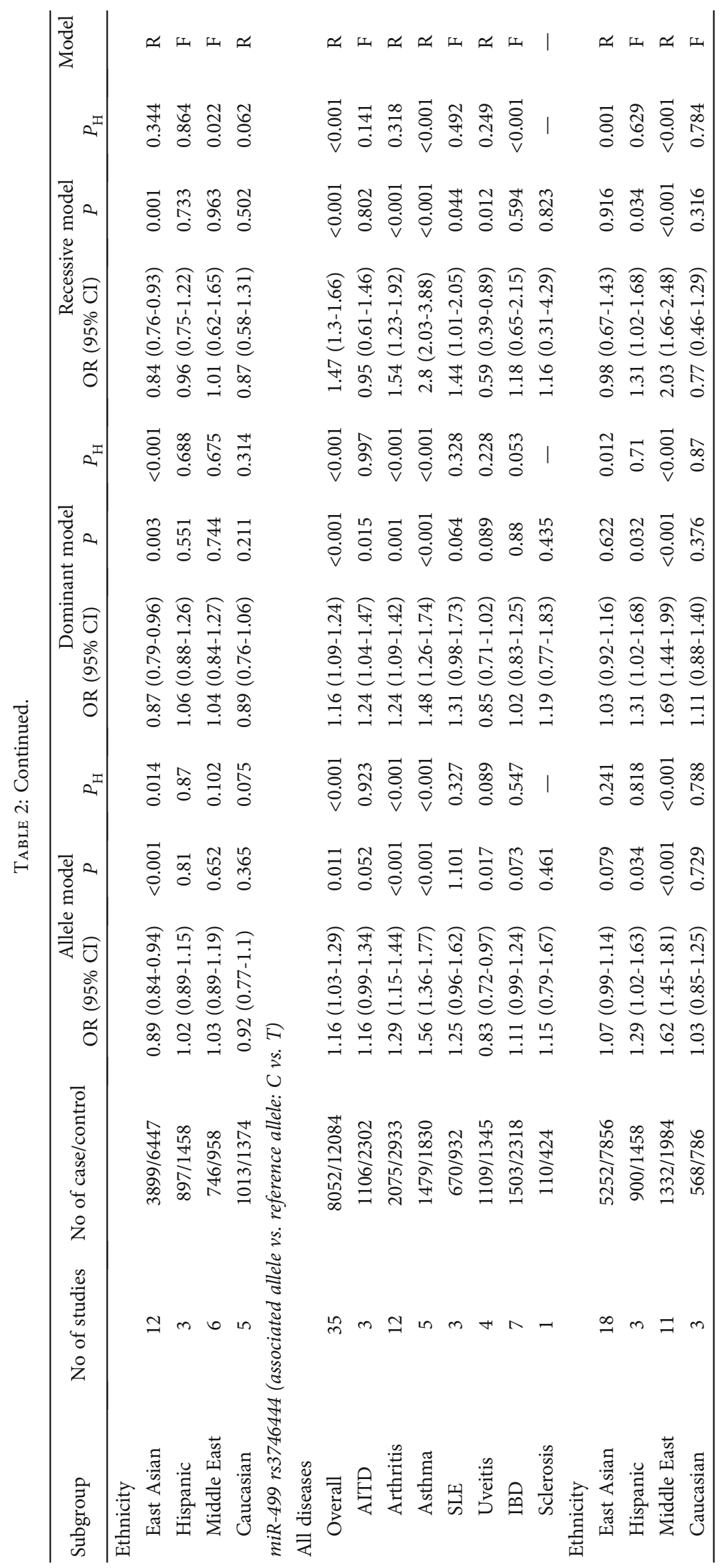


Cai et al.2017

Cai et al.2017

$\mathrm{Xu}$ et al.2015

Niu et al.2015

Hu et al.2017

Su et al.2011

Hoang et al.2017

Jimenez-Morales et al.2012

Jimenez-Morales et al.2012

Jimenez-Morales et al.2012

Oner et al.2015

Yenmis et al.2020

Zhou et al.2014

Zhou et al.2014

Ibrahim et al.2018

Gazouli et al.2013

Gazouli et al.2013

Ciccacci et al.2017

Ciccacci et al.2017

Zhu et al.2016

Zhu et al.2016

Lin et al.2014

Yang et al.2015

Singh et al.2014

Zhang et al.2018

Zha et al.2019

Labib et al.2019

Labib et al.2019

Fenoglio et al.2011

Li et al.2015

Kiselev et al.2019

Papathanasiou et al.2020

Okada et al.2014

Maharaj et al.2018

Maharaj et al.2018

Chatzikyriakidou et al.2010

Zhang et al.2014

Srivastava et al.2016

Hruska et al.2019

Sakoguchi et al.2013

Vreca et al.218

Kaidonis et al.2016

Assmann et al.2017

Zhou et al.2012

Wei et al.2014

Okubo et al.2011

Zhang et al.2011

Avila et al.2017

Avila et al.2017

Avila et al.2017

Lofgren et al.2012

Chatzikyriakidou et al.2010

Shal et al.2013

Shaker et al.2018

Hashemi et al.2013

Yang et al.2011

Zhou et al.2015

Hassine et al.2017

$\mathrm{Hu}$ et al.2019

Ciccacci et al.2016

Ayeldeen et al.2018

Bogunia-Kubik et al.2017

Caputo et al.2020

Caputo et al.2020

Ahmadi et al.2020

Ahmadi et al.2020

Vajargah et al.2018

Wang et al.2020

Overall (I-squared $=80.0 \%, p<0.001$ )

NOTE: weights are from random effects analysis

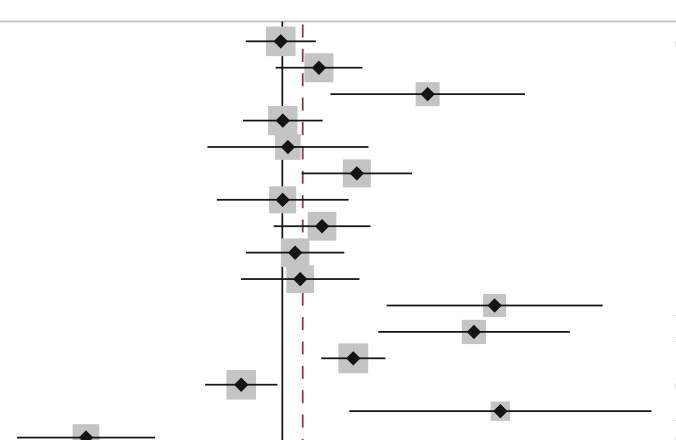

$0.99(0.86,1.15)$

$1.80(1.22,2.68) \quad 1.82$

$1.00(0.85,1.18) \quad 1.86$

$1.02(0.74,1.42) \quad 1.40$

$1.35(1.08,1.69) \quad 1.69$

$1.00(0.77,1.31) \quad 1.57$

$1.17(0.97,1.43) \quad 1.77$

$1.05(0.86,1.29) \quad 1.76$

$1.08(0.85,1.37) \quad 1.65$

$2.37(1.53,3.67) \quad 1.11$

$2.18(1.48,3.21) \quad 1.23$

$1.33(1.17,1.52) \quad 1.93$

$0.85(0.73,0.98) \quad 1.90$

$2.43(1.31,4.48) \quad 0.77$

$0.45(0.34,0.60) \quad 1.53$

$0.82(0.60,1.12) \quad 1.44$

$0.87(0.66,1.14) \quad 1.55$

$0.89(0.66,1.18) \quad 1.51$

$0.78(0.62,0.98) \quad 1.67$

$0.74(0.59,0.93) \quad 1.69$

$0.77(0.65,0.92) \quad 1.83$

$1.18(0.85,1.63) \quad 1.41$

$0.81(0.59,1.12) \quad 1.41$

$0.98(0.83,1.16) \quad 1.84$

$0.86(0.60,1.24) \quad 1.30$

$2.82(1.83,4.33) \quad 1.13$

$2.27(1.50,3.43) \quad 1.17$

$0.87(0.69,1.11) \quad 1.65$

$0.91(0.77,1.08) \quad 1.84$

$0.95(0.66,1.36) \quad 1.32$

$0.92(0.78,1.09) \quad 1.86$

$1.29(0.76,2.21) \quad 0.90$

$1.22(0.60,2.46) \quad 0.64$

$1.90(1.13,3.22) \quad 0.92$

$0.83(0.42,1.64) \quad 0.68$

$1.26(1.07,1.49) \quad 1.84$

$\begin{array}{ll}1.11(0.98,1.26) & 1.95\end{array}$

$0.95(0.73,1.23) \quad 1.59$

$0.94(0.56,1.58) \quad 0.93$

$0.62(0.35,1.08) \quad 0.86$

$0.83(0.68,1.00) \quad 1.78$

$1.55(1.25,1.92) \quad 1.72$

$1.07(0.85,1.34) \quad 1.69$

$1.46(1.26,1.69) \quad 1.89$

$0.83(0.64,1.08) \quad 1.59$

$1.15(0.87,1.52) \quad 1.54$

$0.82(0.68,1.00) \quad 1.77$

$0.88(0.72,1.07) \quad 1.77$

$0.81(0.57,1.15) \quad 1.35$

$0.96(0.84,1.09) \quad 1.93$

$1.04(0.72,1.50) \quad 1.28$

$1.38(1.05,1.81) \quad 1.55$

$1.95(1.32,2.89) \quad 1.23$

$0.93(0.60,1.43) \quad 1.12$

$0.98(0.75,1.29) \quad 1.55$

$1.04(0.89,1.21) \quad 1.89$

$1.47(1.04,2.09) \quad 1.33$

$0.90(0.61,1.34) \quad 1.21$

$1.11(0.83,1.49) \quad 1.50$

$1.95(1.13,3.40) \quad 0.87$

$0.87(0.55,1.36) \quad 1.08$

$1.22(1.00,1.50) \quad 1.76$

$2.08(1.72,2.51) \quad 1.79$

$0.85(0.59,1.22) \quad 1.30$

$1.32(0.81,2.14) \quad 1.00$

$1.34(0.79,2.28) \quad 0.91$

$1.06(0.80,1.40)$

$1.08(1.01,1.15) \quad 100.00$

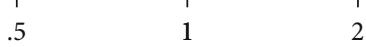

Figure 3: Assessment of the association between miR-146a rs2910164 polymorphism (G vs. C) with AD.

population (allele model, $\mathrm{OR}=0.83,95 \% \mathrm{CI}$ : 0.69-0.99, $P=0.038$; dominant model, $\mathrm{OR}=0.82$, 95\% CI: $0.68-0.99$, $P=0.039)$. Similar results were found in uveitis patients (shown in supplementary materials (available here)).
3.2.2. miR-196a2. Combined results of 26 studies revealed that the T allele of miR-196a2 rs11614913 was associated with a lower risk of $\mathrm{AD}$ (allele model, $\mathrm{OR}=0.92,95 \% \mathrm{CI}$ : $0.88-0.97, P=0.001$, Figure 4; dominant model, $\mathrm{OR}=0.92$, 


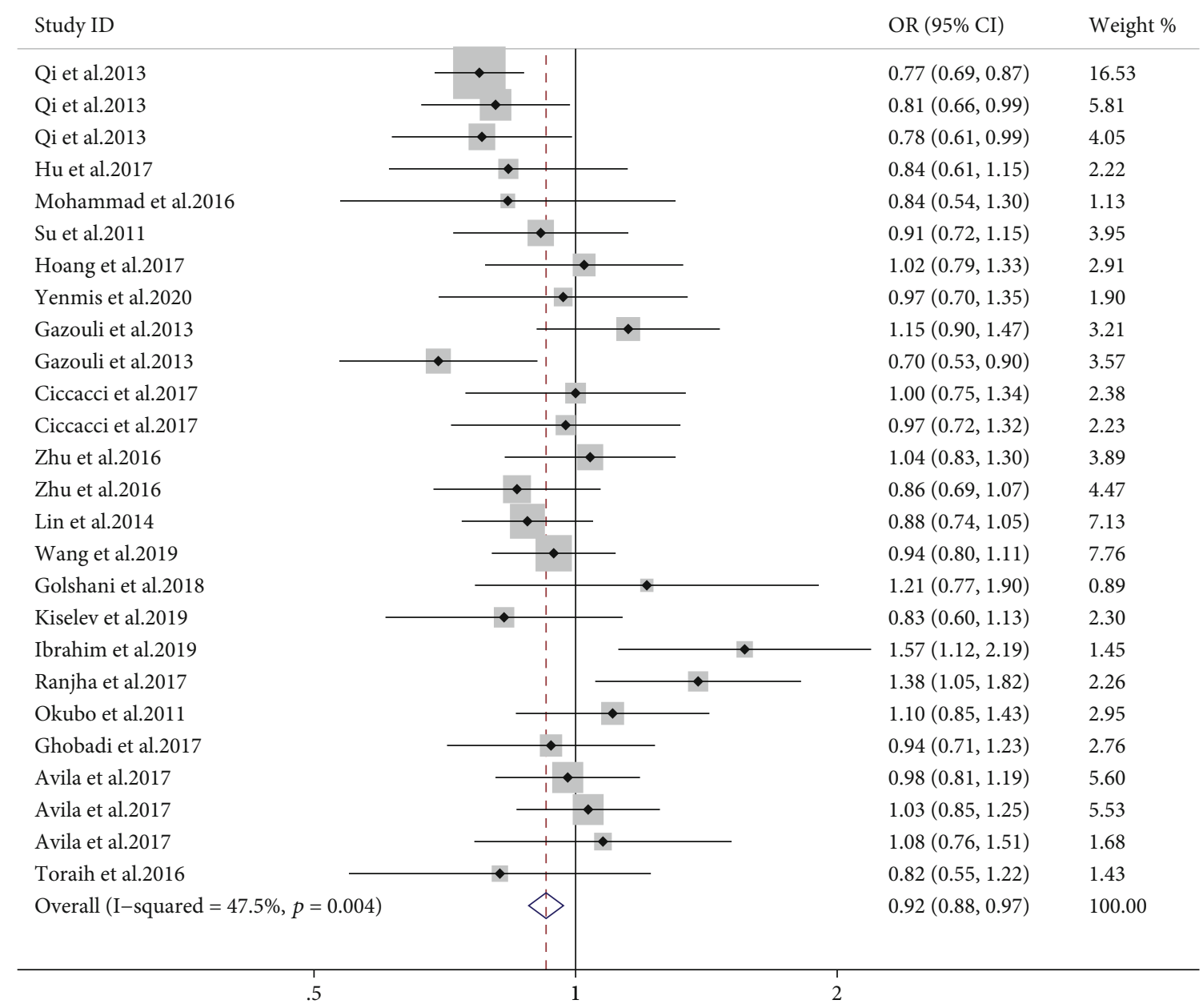

FIGURE 4: Estimation of the association between miR-196a2 rs11614913 polymorphism ( $\mathrm{T}$ vs. C) with AD.

95\% CI: $0.86-0.98, P=0.017$; recessive model, $\mathrm{OR}=0.87$, 95\% CI: $0.81-0.95, P=0.002)$. Furthermore, there was a reduced risk of uveitis in the three genetic models (allele model, OR $=0.80,95 \% \mathrm{CI}: 0.73-0.87, P<0.001$; dominant model, $\mathrm{OR}=0.74,95 \% \mathrm{CI}: 0.64-0.84, P<0.001$; recessive model, $\mathrm{OR}=0.74,95 \% \mathrm{CI}: 0.62-0.87, P<0.001$ ), but an increased risk of T1DM in the allele and recessive model. The stratified analysis results demonstrated that rs11614913 $\mathrm{T}$ was significantly related with a decreased risk of $\mathrm{AD}$ in the East Asian population (shown in supplementary materials (available here)). Subgroup meta-analysis by the NOS scale revealed a significant negative association in the high-quality studies $(\mathrm{OR}=0.90,95 \% \mathrm{CI}$ : $0.86-0.95, P<0.001)$ but not the low-quality studies (shown in supplementary materials (available here)).

3.2.3. $m i R-499$. Meta-analysis of 35 case-control studies showed miR-499 rs3746444 is a predisposing cause of $\mathrm{AD}$ (allele model, $\mathrm{OR}=1.16$, 95\% CI: $1.03-1.29, P=0.011$, Figure 5; dominant model, $\mathrm{OR}=1.16,95 \% \mathrm{CI}: 1.09-1.24$, $P<0.001$; recessive model, $\mathrm{OR}=1.47,95 \% \mathrm{CI}: 1.30-1.66$, $P<0.001)$. In the subgroup analysis by disease subtypes, rs3746444 polymorphisms increased susceptibility for arthritis and asthma (allele model, OR $=1.29$, 95\% CI:
1.15-1.44, $\quad P<0.001 ;$ dominant model, $\mathrm{OR}=1.24,95 \%$ CI: $1.09-1.42, P=0.001$; recessive model, $\mathrm{OR}=1.54,95 \%$ CI: $1.23-1.92, P<0.001$; allele model, $\mathrm{OR}=1.56,95 \% \mathrm{CI}$ : 1.36-1.77, $P<0.001$; dominant model, $\mathrm{OR}=1.48,95 \%$ CI: $1.26-1.74, P<0.001$; recessive model, $\mathrm{OR}=2.80,95 \%$ CI:2.03-3.88, $P<0.001$, respectively). On the other hand, a reduced susceptibility was observed for uveitis (allele model, $\mathrm{OR}=0.83,95 \% \mathrm{CI}: 0.72-0.97, P=0.017$; recessive model, $\quad \mathrm{OR}=0.59, \quad 95 \% \quad \mathrm{CI}: \quad 0.39-0.89, \quad P=0.012) . \quad \mathrm{A}$ stratified analysis hinted that rs3746444 $\mathrm{C}$ delivered an increased risk of $\mathrm{AD}$ in the Hispanic and Middle East region (shown in supplementary materials (available here)). Additionally, subgroup meta-analysis based on the NOS scale revealed a significant positive association in the high-quality studies $(\mathrm{OR}=1.21,95 \% \mathrm{CI}: 1.03-1.41, P=0.018)$ but not the low-quality studies (shown in supplementary materials (available here)).

3.2.4. Other miRNAs. In addition, a significantly increased risk was observed between miR-149 rs2292832/miR-27a rs895819/miR-182 rs76481776/miR-23a rs3745453 and AD susceptibility in the overall population (allele model, $\mathrm{OR}=1.15,95 \% \mathrm{CI}: 1.06-1.24, P=0.001$; dominant model, $\mathrm{OR}=1.13,95 \% \mathrm{CI}: 1.01-1.26, \quad P=0.027$; allele model, 


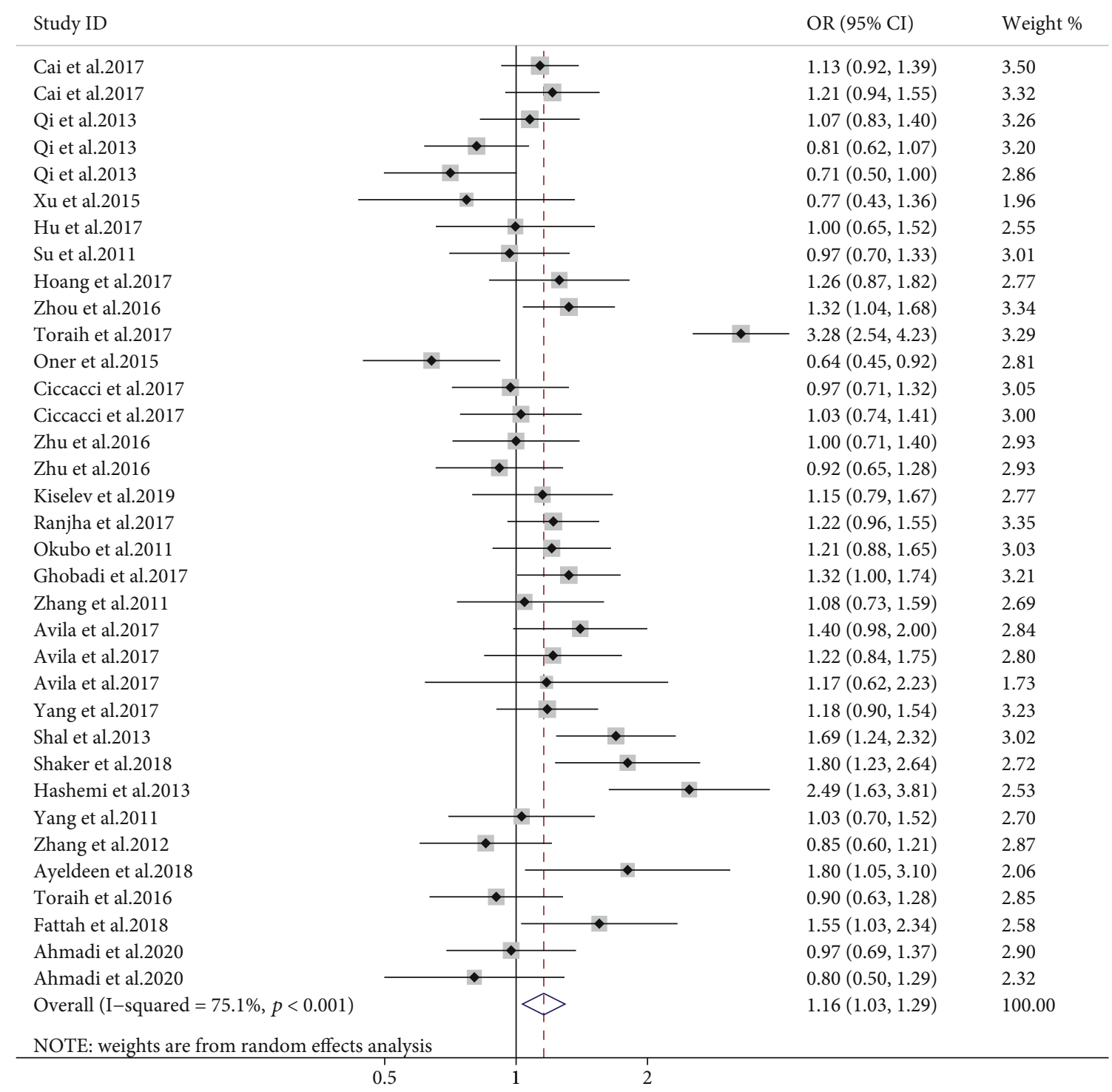

FIGURE 5: Evaluation of the association between miR-499 rs3746444 polymorphism (C vs. T) with AD.

$\mathrm{OR}=1.11,95 \% \mathrm{CI}: 1.01-1.22, P=0.043$; recessive model, $\mathrm{OR}$ $=1.28,95 \%$ CI:1.05-1.55, $P=0.013$; allele model, $\mathrm{OR}=1.62$, 95\% CI: 1.43-1.82, $P<0.001$; dominant model, $\mathrm{OR}=1.63$, 95\% CI: 1.43-1.86, $P<0.001$; recessive model, $\mathrm{OR}=2.22,95 \%$ CI: $1.48-3.34, P<0.001$; allele model, $\mathrm{OR}=1.68,95 \% \mathrm{CI}$ : $1.39-2.05, P<0.001$; dominant model, $\mathrm{OR}=1.61,95 \%$ CI: $1.25-2.08, \quad P<0.001$; recessive model, $\mathrm{OR}=2.82,95 \%$ CI: $1.84-4.32, P<0.001$, respectively). Subgroup analysis showed an increased risk of arthritis and asthma but a reduced risk of IBD with rs2292832 polymorphisms. Moreover, there was no apparent correlation between other miRNAs and $\mathrm{AD}$ susceptibility (shown in supplementary materials (available here)).

3.3. Heterogeneity Test and Meta-regression Analysis. The merged results revealed conspicuous heterogeneities in the combined disease groups (heterogeneity: $I^{2}=47.5 \%-76.3 \%$, $P<0.001$, shown in Table 2 and supplementary materials (available here)). To further explore the source of heterogeneity, we performed a univariate metaregression analysis based on the random effects model. Several covariate factors such as disease subtype, genotypic method, ethnicity, mean age, the number of cases, and percentage of females in cases were evaluated using three genetic models (allelic, dominant, and recessive, Table 3 ). In our combined analysis, a statistically significant effect on the summary ORs through disease subtype and ethnicity was discovered (allele model, $P=0.031, \quad$ OR $=1.02-1.48 ; \quad P=0.005, \quad \mathrm{OR}=1.25-3.42)$, which could partly account for the variation of heterogeneity (adjusted $R^{2}=35.16 \%$, adjusted $R^{2}=45.71 \%$, respectively) (shown in Table 3 ).

3.4. Sensitivity Analysis. To evaluate the effect of publication on the robustness of our pooled effect estimate, a sensitivity analysis was performed by deleting each study once at a time in the three genetic models. As a result, the summary OR did 
TABLE 3: Metaregression for the heterogeneity of miR-146a rs2910164 comparison in our meta-analysis.

\begin{tabular}{lccccccccccccc}
\hline \multirow{2}{*}{ Covariate factors } & \multicolumn{4}{c}{ Allele model } & \multicolumn{4}{c}{ Dominant model } & \multicolumn{4}{c}{ Recessive model } \\
& Exp $(b)$ & Std. Err & $P$ value & $95 \%$ CI & Exp $(b)$ & Std. Err & $P$ value & 95\% CI & Exp $(b)$ & Std. Err & $P$ value & $95 \%$ CI \\
\hline Disease subtype & 1.23 & 0.09 & 0.031 & $1.02-1.48$ & 1.52 & 0.24 & 0.015 & $1.09-1.23$ & 1.53 & 0.24 & 0.017 & $1.02-1.36$ \\
Genotypic method & 0.93 & 0.08 & 0.377 & $0.78-1.09$ & 1.02 & 0.02 & 0.388 & $0.98-1.05$ & 1.02 & 0.02 & 0.321 & $0.97-1.06$ \\
Ethnicity & 2.51 & 0.74 & 0.005 & $1.25-3.42$ & 1.09 & 0.09 & 0.264 & $0.93-1.29$ & 1.19 & 0.100 & 0.043 & $1.01-1.41$ \\
Mean age & 1.22 & 0.14 & 0.083 & $0.97-1.55$ & 1.12 & 0.17 & 0.449 & $0.83-1.51$ & 1.13 & 0.17 & 0.446 & $0.83-1.53$ \\
Number of cases & 0.96 & 0.07 & 0.589 & $0.81-1.13$ & 0.99 & 0.03 & 0.909 & $0.94-1.06$ & 1.02 & 0.04 & 0.655 & $0.94-1.11$ \\
Percentage of female & 1.04 & 0.11 & 0.736 & $0.84-1.28$ & 0.95 & 0.14 & 0.736 & $0.72-1.27$ & 1.11 & 0.17 & 0.495 & $0.82-1.51$ \\
\hline
\end{tabular}

Exp (b): odds ratio; Std. Err: standard error; CI: confidence interval.

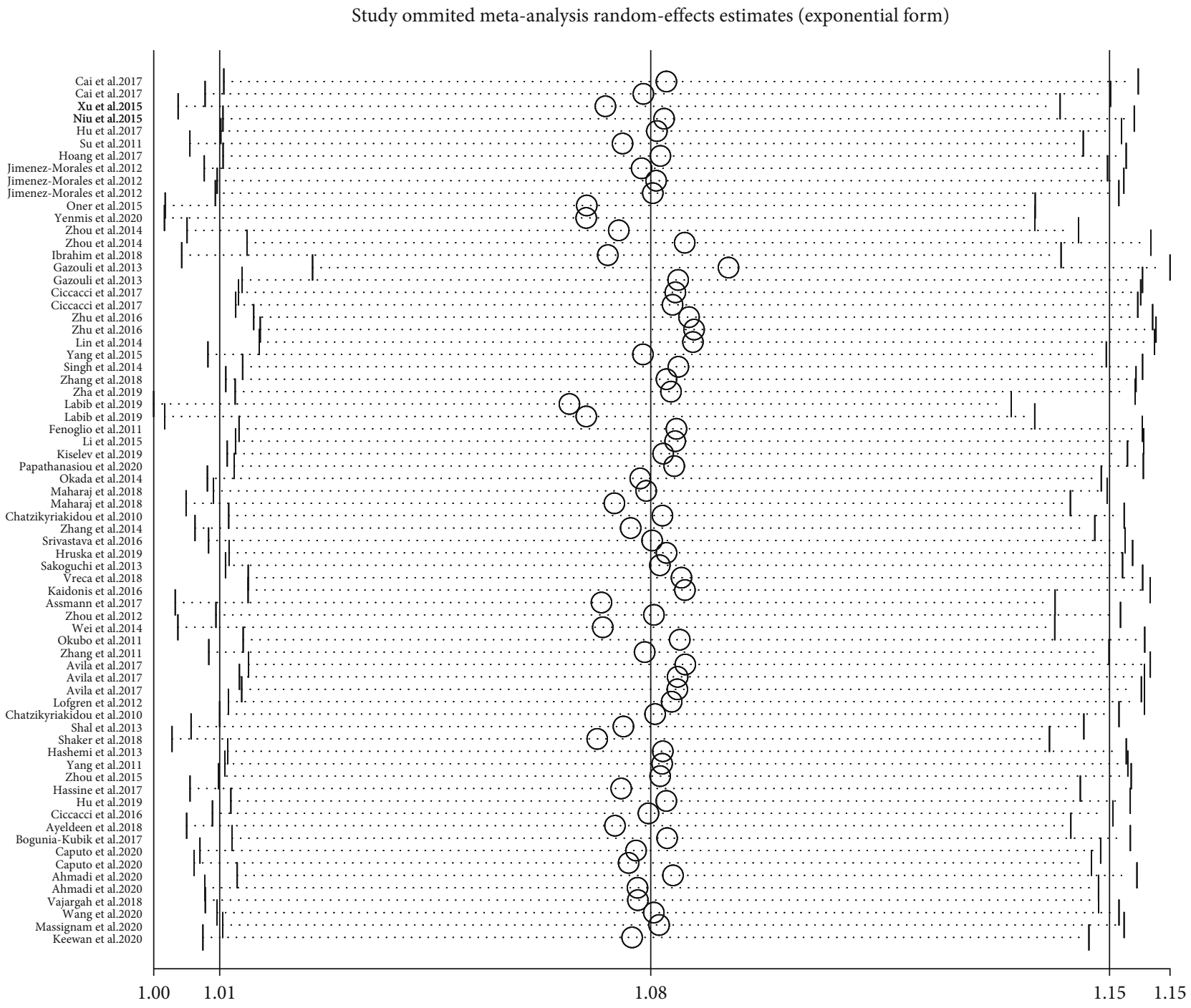

FIgURE 6: Assessment of the sensitivity analysis between miR-146a rs2910164 polymorphism (G vs. C) with AD.

not make any difference on the overall risk estimates, which suggested that our meta-analysis was reliable (Figure 6, rs2910164 G vs. C).

3.5. Publication Bias. Publication bias was investigated according to the test of Begg's funnel plot and Egger's regres- sion analysis. Due to the relatively small number of included studies, publication bias analysis could not be carried out for miR-155 rs767649, miR-125a rs12976445, miR-182 rs76481776, miR-585 rs62376935, miR-23a rs3745453, miR106a rs3747440, miR-122 rs17669, miR-124a rs531564, and miR-137 rs1625579. As for the other miRNA polymorphisms, 
no evidence of publication bias was observed neither with the Begg's funnel plot nor the Egger's test (Figure 7, rs2910164 G vs. C), which implies that our results were statistically robust $(P>0.05$, Table 4$)$.

\section{Discussion}

In this systematic meta-analysis of 87 case-control studies, 17 SNPs from 14 miRNA genes were shown to be associated with the susceptibility to AD. Five miRNAs with eight variants were shared across $\mathrm{AD}$ and may play potential roles in the pathogenesis of $\mathrm{AD}$ (shown in supplementary materials (available here)).

A mounting body of evidence has demonstrated that miRNAs cause gene silencing by degrading targeted mRNAs or by inhibiting translation. Common variants in miRNAs can rearrange a broad range of biological processes by influencing the processing or altering target selection of miRNAs [109], thus dysregulating miRNA expression, which may be involved in the development of a wide range of diseases including AD. Although several meta-analysis studies have shown the association between miRNA variants with $\mathrm{AD}$, most of them only discussed a single SNP.

Previous studies have demonstrated that miRNAs may play an important role in the regulation of the immune system. The well-known miR-146a, which is a negative regulator of the NF- $\kappa \mathrm{B}$ activation pathway, can modulate mRNAs that encode proteins involved in the control of innate or adaptive immune responses [110]. SNP rs2910164, which is located in the stem sequence of the miR-146a precursor, can directly influence the expression of miR-146a [111]. Five studies presented independent evidence that the rs2910164 $\mathrm{G}$ allele was not correlated with arthritis [24-26, 112, 113]. Furthermore, a previous meta-analysis [108] also showed no association with inflammatory arthritis, IBD, and a uveitis subgroup. Our meta-analysis, however, did suggest that the $\mathrm{G}$ allele was protective against IBD and that it was a risk factor for arthritis, asthma, uveitis, and psoriasis. The remarkable difference between our data and the earlier meta-analyses may be due to the fact that we included more studies and therefore had a higher number of patients. rs57095329, which is located in the promoter region of the miR-146a gene, has been shown to induce the expression of miR-146a by altering its binding affinity with Ets-1 [62]. Furthermore, individuals containing the risk $\mathrm{G}$ allele tended to express a lower level of miR-146a in Asian patients, and further functional studies showed that it was a negative regulator of the IFN pathway. Our meta-analysis confirmed earlier studies $[112,113]$, showing that rs57095329 G was a high-risk factor for SLE but not for other AD in East Asian regions. These pooled results could be explained by the disease-specific influence on SLE. Moreover, recent SLE GWASs have identified the disease-related SNP-rs2431697, which lies upstream of the miR-146a gene, and showed that the $\mathrm{C}$ allele conferred protective susceptibility to SLE in Asians and Caucasians $[114,115]$. Compared with a metaanalysis performed by earlier by others [112], our metaanalysis included more studies and a larger sample size and indicated that the $\mathrm{C}$ allele was protective against SLE

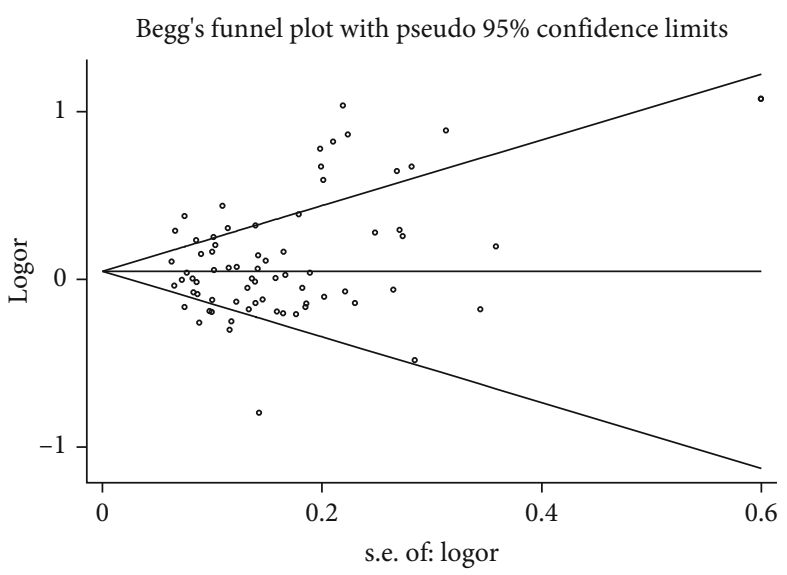

Figure 7: Estimation of the publication bias between miR-146a rs2910164 polymorphism (G vs. C) with AD.

in Asians and Caucasians, whereas the latter study was confined to an Asian population.

miR-146a rs6864584, which is positioned in the miR146a precursor promoter region, also affects the expression of miR-146a [62]. Recent studies could not detect an association between rs6864584 $\mathrm{C} / \mathrm{T}$ and $\mathrm{KD}$ or asthma [44, 63]. Our meta-analysis is the first to confirm the association between rs6864584 and uveitis.

The transcript variant (rs11614913) of miR-196a2 precursor has been reported to influence the efficient processing of mature miR-196a 2 and the expression of its target gene [116]. Our analysis showed that the $\mathrm{T}$ allele conferred protection against uveitis. A decreased risk of miR-196a2 variants has been reported with T1DM in children and adolescents, but rs11614913 genotypes were not shown to affect miRNA expression [79]. An allele mutation of rs11614913 from $\mathrm{C}$ to $\mathrm{T}$ was detected in a majority of glioma tissues, but no association was discovered with the genotype [117]. In other words, the differential expression of miR-196a2 was probably not mediated by rs11614913 itself but by other factors.

Studies have confirmed that miR-499 rs3746444 is located in the pre-miRNA region and influences the binding of target mRNAs to $3 \mathrm{p}$ mature miRNA [118]. mir-499 targets the IL-17 receptor B, IL-6, and other cytokines, all of which play an important role in the pathogenesis of RA [119]. An miR-499 mutation, rs3746444, was shown to be associated with RA as well as with disease severity in Egyptian patients [43]. The CC genotype and C allele of this variant also confer genetic predisposition to RA in Iranians. The association between miR-499 polymorphisms and RA could not be confirmed in Chinese patients [72]. Our combined results not only showed that the rs3746444 C allele is associated with an increased risk for arthritis but also showed that it was protective for uveitis in Middle East populations. In agreement with a previous meta-analysis [27], we also showed an association between the CC genotype and asthma.

The rs2292832 T>C mutation of miR-149 may affect its expression and susceptibility to disease $[40,63]$. miR-149 is a proapoptotic miRNA that affects the expression of the Akt1 
TABLE 4: Bias between miRNA polymorphisms with autoimmune diseases in our meta-analysis.

\begin{tabular}{|c|c|c|c|c|c|c|c|}
\hline \multirow{2}{*}{ miRNA polymorphisms } & \multirow{2}{*}{ Number of publications } & \multicolumn{2}{|c|}{ Allele model } & \multicolumn{2}{|c|}{ Dominant model } & \multicolumn{2}{|c|}{ Recessive model } \\
\hline & & Begg's test & Egger's test & Begg's test & Egger's test & Begg's test & Egger's test \\
\hline miR-146a rs2910164 & 70 & 0.055 & 0.286 & 0.124 & 0.218 & 0.235 & 0.056 \\
\hline miR-196a2 rs11614913 & 26 & 0.343 & 0.061 & 0.427 & 0.084 & 0.930 & 0.948 \\
\hline miR-499 rs3746444 & 35 & 0.680 & 0.432 & 0.910 & 0.910 & 0.234 & 0.239 \\
\hline miR-146a rs57095329 & 18 & 0.544 & 0.594 & 0.443 & 0.608 & 0.155 & 0.825 \\
\hline miR-146a rs2431697 & 8 & 0.536 & 0.498 & 0.734 & 0.505 & 0.308 & 0.057 \\
\hline miR-146a rs6864584 & 5 & 0.142 & 0.123 & 0.221 & 0.120 & 0.462 & 0.302 \\
\hline miR-149 rs2292832 & 9 & 0.404 & 0.052 & 0.251 & 0.213 & 0.754 & 0.820 \\
\hline miR-27a rs895819 & 6 & 0.188 & 0.453 & 0.707 & 0.794 & 0.452 & 0.214 \\
\hline
\end{tabular}

and E2F1 gene, which has been shown to promote cell growth and cell cycle progression in IBD-associated colorectal cancer [120]. Our meta-analysis revealed significant associations between rs2292832 and the risk of developing arthritis, asthma, and IBD. The available data was focused on Asian populations, and it would be interesting to investigate a possible association between miR-149 variants and $\mathrm{AD}$ in other ethnic groups.

We did not find associations for several miRNA variants including miR-27a, miR-155, miR-125a, miR-182, miR-585, miR-23a, miR-106a, miR-122, miR-124a, and miR-137. Our analysis did reveal an association between miR-182 rs76481776 and uveitis susceptibility as well as an association between miR-23a rs3745453 and sclerosis.

Understanding the specific miRNA-regulated genetic networks and molecular mechanisms by which miRNAs participate in the immune system is a promising area of research and promotes their clinical application. The diagnostic and therapeutic manners of miRNAs have long been acknowledged, which are regarded as clinical biomarkers for monitoring disease evolution during treatment [121]. In the future, miRNAs in biofluids such as saliva could be excellent biomarkers, because their collection is noninvasive and easy to be performed [122]. Thus, it is urgent to explore the molecular role of miRNAs in the pathophysiology of AD and to evaluate possible clinical and future implications for a personalized approach.

Although we retrieved all current available studies, some limitations of our analysis should be mentioned. First, the disproportionate numbers of cases (range: $0.11 \%-32.58 \%$ ) in different $\mathrm{AD}$ subtypes might have yielded different sample sizes; thus, the statistical power may show potential heterogeneity. Second, several studies were mainly focused on asthma and Kawasaki disease risk relationship with younger children (younger than five years old), and this may inevitably produce age bias. Third, several groups only contained two studies, which makes it difficult to generalize results, suggesting that larger sample sizes are needed to validate the relationship. Fourth, the current research should be registered in the PROSPERO or Cochrane system, and we hope to do so in future but for the time being would like to mention that our meta-analysis was performed strictly in accordance with the process of systematic review. In addition, the association level identified by current studies was low because of imprecision according to GRADE profiler. Finally, more attention should be made concerning a possible gender bias. However, metaregression did not show that the gender ratio affected our pooled results.

\section{Conclusions}

Taken together, our meta-analysis provides evidence that miR-146a, miR-196a2, miR-499, miR-149, and miR-27a polymorphisms are associated with $\mathrm{AD}$ susceptibility. Some polymorphisms are shared by several $\mathrm{AD}$ in certain ethnic groups and/or geographic locations. Some miRNA polymorphisms show protection in some diseases and an increased susceptibility in others. These results provide further support to the complexity of autoimmune disease and suggest that prevention and treatment should be tailored for each specific immune disorder.

\section{Data Availability}

The data that support the findings of this study are available from the corresponding author upon reasonable request.

\section{Ethical Approval}

All analyses were based on previously published studies; thus, no ethical approval and patient consent are required.

\section{Conflicts of Interest}

The authors declare that they have no conflicts of interest to disclose.

\section{Authors' Contributions}

P.Y. conceived the study. J.Z. and H.T. performed and checked the available information from eligible articles. J.Z., Q.C., and G.S. analyzed the data. Q.C. prepared the Figures 1-7 and Tables 1-4. J.Z. wrote the main manuscript text. P.Y. reviewed and revised the manuscript. All authors read and approved the final manuscript. 


\section{Acknowledgments}

This work was supported by the Natural Science Foundation Major International (Regional) Joint Research Project (81720108009), the National Natural Science Foundation Key Program (81930023), the Chongqing Outstanding Scientists Project (2019), the Chongqing Chief Medical Scientist Project (2018), the Chongqing Key Laboratory of Ophthalmology (CSTC, 2008CA5003), and the Chongqing Science \& Technology Platform and Base Construction Program (cstc2014pt-sy10002).

\section{Supplementary Materials}

Supplement Table 1: PRISMA 2009 Checklist. Supplement Table 2: meta-analysis of other miRNA-SNPs with autoimmune diseases. Supplement Table 3: the most important findings of the meta-analysis. (Supplementary Materials)

\section{References}

[1] P. Marrack, J. Kappler, and B. L. Kotzin, "Autoimmune disease: why and where it occurs," Nature Medicine, vol. 7, no. 8, pp. 899-905, 2001.

[2] K. Hemminki, W. Huang, J. Sundquist, K. Sundquist, and J. Ji, “Autoimmune diseases and hematological malignancies: exploring the underlying mechanisms from epidemiological evidence," Seminars in Cancer Biology, vol. 64, pp. 114-121, 2020.

[3] M. K. Desai and R. D. Brinton, "Autoimmune disease in women: endocrine transition and risk across the lifespan," Frontiers in Endocrinology, vol. 10, p. 265, 2019.

[4] X. Hu and M. Daly, "What have we learned from six years of GWAS in autoimmune diseases, and what is next?," Current Opinion in Immunology, vol. 24, no. 5, pp. 571-575, 2012.

[5] G. R. Burmester and J. E. Pope, "Novel treatment strategies in rheumatoid arthritis," Lancet, vol. 389, no. 10086, pp. 2338$2348,2017$.

[6] C. Fenoglio, C. Cantoni, M. de Riz et al., "Expression and genetic analysis of miRNAs involved in CD4+ cell activation in patients with multiple sclerosis," Neuroscience Letters, vol. 504, no. 1, pp. 9-12, 2011.

[7] S. Jimenez-Morales, R. Gamboa-Becerra, V. Baca et al., "MiR146a polymorphism is associated with asthma but not with systemic lupus erythematosus and juvenile rheumatoid arthritis in Mexican patients," Tissue Antigens, vol. 80, no. 4, pp. 317-321, 2012.

[8] W. Zhang, X. Yi, S. Guo et al., "A single-nucleotide polymorphism of miR-146a and psoriasis: an association and functional study," Journal of Cellular and Molecular Medicine, vol. 18, no. 11, pp. 2225-2234, 2014.

[9] Z. Niu, J. Wang, H. Zou, C. Yang, W. Huang, and L. Jin, "Common MIR146A polymorphisms in Chinese ankylosing spondylitis subjects and controls," PLoS One, vol. 10, no. 9, article e0137770, 2015.

[10] J. Wang, J. Li, H. Qiu et al., "Association between miRNA$196 a 2$ rs11614913 T\&gt;C polymorphism and Kawasaki disease susceptibility in southern Chinese children," Journal of Clinical Laboratory Analysis, vol. 33, no. 7, article e22925, 2019.
[11] I. Papathanasiou, E. Mourmoura, C. Balis, and A. Tsezou, "Impact of miR-SNP rs2910164 on miR-146a expression in osteoarthritic chondrocytes," Advances in Medical Sciences, vol. 65, no. 1, pp. 78-85, 2020.

[12] M. A. Senousy, O. G. Shaker, N. H. Sayed, N. Fathy, and M. A. Kortam, "LncRNA GAS5 and miR-137 polymorphisms and expression are associated with multiple sclerosis risk: mechanistic insights and potential clinical impact," ACS Chemical Neuroscience, vol. 11, no. 11, pp. 1651-1660, 2020.

[13] G. Yenmis, T. Soydas, C. G. Ekmekci, A. C. Y. Guvercin, O. S. Kucuk, and G. K. Sultuybek, "Fas and microRNAs Variations as a Possible Risk for Behçet Disease," JCR: Journal of Clinical Rheumatology, vol. Publish Ahead of Print, 2020.

[14] H. Y. Xu, Z. Y. Wang, J. F. Chen et al., "Association between ankylosing spondylitis and the miR-146a and miR-499 polymorphisms," PLoS One, vol. 10, no. 4, article e0122055, 2015.

[15] M. Hashemi, E. Eskandari-Nasab, Z. Zakeri et al., "Association of pre-miRNA-146a rs2910164 and premiRNA-499 rs3746444 polymorphisms and susceptibility to rheumatoid arthritis," Molecular Medicine Reports, vol. 7, no. 1, pp. 287-291, 2013.

[16] C. Xiao and K. Rajewsky, "MicroRNA control in the immune system: basic principles," Cell, vol. 136, no. 1, pp. 26-36, 2009.

[17] T. Y. Ha, "The role of microRNAs in regulatory $\mathrm{T}$ cells and in the immune response," Immune Netw, vol. 11, no. 1, pp. 11-41, 2011.

[18] G. Evangelatos, G. E. Fragoulis, V. Koulouri, and G. I. Lambrou, "MicroRNAs in rheumatoid arthritis: from pathogenesis to clinical impact," Autoimmunity Reviews, vol. 18, no. 11, p. 102391, 2019.

[19] H. Mohammadi, M. Hemmatzadeh, F. Babaie et al., "MicroRNA implications in the etiopathogenesis of ankylosing spondylitis," Journal of Cellular Physiology, vol. 233, no. 8, pp. 5564-5573, 2018.

[20] R. S. Pillai, S. N. Bhattacharyya, C. G. Artus et al., "Inhibition of translational initiation by Let-7 microRNA in human cells," Science, vol. 309, no. 5740, pp. 1573-1576, 2005.

[21] D. T. Humphreys, B. J. Westman, D. I. Martin, and T. Preiss, "MicroRNAs control translation initiation by inhibiting eukaryotic initiation factor $4 \mathrm{E} / \mathrm{cap}$ and poly (A) tail function," Proceedings of the National Academy of Sciences of the United States of America, vol. 102, no. 47, pp. 16961-16966, 2005.

[22] S. Nottrott, M. J. Simard, and J. D. Richter, "Human let-7a miRNA blocks protein production on actively translating polyribosomes," Nature Structural \& Molecular Biology, vol. 13, no. 12, pp. 1108-1114, 2006.

[23] C. P. Petersen, M. E. Bordeleau, J. Pelletier, and P. A. Sharp, "Short RNAs repress translation after initiation in mammalian cells,” Molecular Cell, vol. 21, no. 4, pp. 533-542, 2006.

[24] H. F. Chen, T. T. Hu, X. Y. Zheng et al., “Association between miR-146a rs2910164 polymorphism and autoimmune diseases susceptibility: a meta-analysis," Gene, vol. 521, no. 2, pp. 259-264, 2013.

[25] D. Wang and G. Pan, "Association of rs2910164 polymorphism in miRNA-146 and rs3746444 polymorphism in miRNA-499 with inflammatory arthritis: a meta-analysis," BioMed Research International, vol. 2019, Article ID 7305750, 9 pages, 2019.

[26] K. Li, H. Tie, N. Hu et al., "Association of two polymorphisms rs2910164 in miRNA-146a and rs3746444 in miRNA-499 
with rheumatoid arthritis: a meta-analysis," Human Immunology, vol. 75, no. 7, pp. 602-608, 2014.

[27] J. Dong, D. Sun, and F. Lu, "Association of two polymorphisms of miRNA-146a rs2910164 (G > C) and miRNA499 rs3746444 ( $\mathrm{T}>\mathrm{C}$ ) with asthma: a meta-analysis," The Journal of Asthma, vol. 58, no. 8, pp. 995-1002, 2021.

[28] J. R. Landis and G. G. Koch, "The measurement of observer agreement for categorical data," Biometrics, vol. 33, no. 1, pp. 159-174, 1977.

[29] D. Atkins, D. Best, P. A. Briss et al., "Grading quality of evidence and strength of recommendations," BMJ, vol. 328, no. 7454, p. 1490, 2004.

[30] J. P. Higgins, S. G. Thompson, J. J. Deeks, and D. G. Altman, "Measuring inconsistency in meta-analyses," BMJ, vol. 327, no. 7414, pp. 557-560, 2003.

[31] Z. M. Tang, P. Wang, P. P. Chang et al., "Association between rs2431697 T allele on 5q33.3 and systemic lupus erythematosus: case-control study and meta-analysis," Clinical Rheumatology, vol. 34, no. 11, pp. 1893-1902, 2015.

[32] P. Hruska, D. Kuruczova, V. Vasku, and J. Bienertova-Vasku, "MiR-21 binding site SNP within ITGAM associated with psoriasis susceptibility in women," PLoS One, vol. 14, no. 6, article e0218323, 2019.

[33] D. Che, J. Li, L. Fu et al., "The rs1625579 T\&gt;G polymorphism in the $<$ em $>$ miRNA-137</em $>$ gene confers a risk of early-onset Kawasaki disease in a southern Chinese population," Infect Drug Resist, vol. Volume 11, pp. 1055-1060, 2018.

[34] Y. J. Sheng, J. H. Xu, Y. G. Wu et al., "Association analyses confirm five susceptibility loci for systemic lupus erythematosus in the Han Chinese population," Arthritis Research \& Therapy, vol. 17, no. 1, p. 85, 2015.

[35] X. K. Yang, P. Li, C. Zhang et al., "Association between IRAK1 rs3027898 and miRNA-499 rs3746444 polymorphisms and rheumatoid arthritis: a case control study and meta-analysis," Zeitschrift für Rheumatologie, vol. 76, no. 7, pp. 622-629, 2017.

[36] M. Zhu, D. Li, M. Jin, and M. Li, “Association between microRNA polymorphisms and the risk of inflammatory bowel disease," Molecular Medicine Reports, vol. 13, no. 6, pp. 5297-5308, 2016.

[37] O. G. Shaker, O. O. Abdelaleem, N. A. Fouad et al., "Association BetweenmiR-155, its polymorphism and ischemiamodified albumin in patients with rheumatoid arthritis," Journal of Interferon \& Cytokine Research, vol. 39, no. 7, pp. 428-437, 2019.

[38] D. A. Labib, O. G. Shaker, R. M. El Refai, S. A. Ghoniem, and A. Elmazny, "Association betweenmiRNA-146aand polymorphisms of its target Gene,IRAK1, regarding susceptibility to and clinical features of systemic lupus erythematous and multiple sclerosis," Laboratoriums Medizin, vol. 50, no. 1, pp. 34-41, 2019.

[39] X. W. Su, Y. Yang, M. L. Lv et al., "Association between single-nucleotide polymorphisms in pre-miRNAs and the risk of asthma in a Chinese population," DNA and Cell Biology, vol. 30, no. 11, pp. 919-923, 2011.

[40] J. Li, J. Wang, X. Su et al., “Association between the miRNA149 rs2292832 T>C polymorphism and Kawasaki disease susceptibility in a southern Chinese population," Journal of Clinical Laboratory Analysis, vol. 34, no. 4, article e23125, 2020.
[41] Y. Takuse, M. Watanabe, N. Inoue et al., "Association of IL-10-regulating microRNAs in peripheral blood mononuclear cells with the pathogenesis of autoimmune thyroid disease," Immunological Investigations, vol. 46, no. 6, pp. 590-602, 2017.

[42] S. Singh, G. Rai, and A. Aggarwal, "Association of microRNA-146a and its target gene IRAK1 polymorphism with enthesitis related arthritis category of juvenile idiopathic arthritis," Rheumatology International, vol. 34, no. 10, pp. 1395-1400, 2014.

[43] A. S. el-Shal, N. M. Aly, S. M. Galil, M. A. Moustafa, and W. A. Kandel, “Association of_microRNAs_genes polymorphisms with rheumatoid arthritis in Egyptian female patients," Joint, Bone, Spine, vol. 80, no. 6, pp. 626-631, 2013.

[44] L. Zha, S. Li, X. Liu et al., "Association of miR-146a gene polymorphism at loci rs2910164 G/C, rs57095329 A/G, and rs6864584 T/C with susceptibility to Kawasaki disease in Chinese children," Pediatric Cardiology, vol. 40, no. 3, pp. 504-512, 2019.

[45] J. Lin, Y. Huang, X. Zhang, J. Chen, and H. Sheng, "Association of miR-146a rs2910164 with childhood IgA nephropathy," Pediatric Nephrology, vol. 29, no. 10, pp. 1979-1986, 2014.

[46] O. O. Abdelaleem, N. A. Fouad, O. G. Shaker et al., "Association of miR-146a rs57095329 with Behçet's disease and its complications," British Journal of Biomedical Science, vol. 78, no. 2, pp. 63-66, 2021.

[47] R. Ranjha, N. K. Meena, A. Singh, V. Ahuja, and J. Paul, "Association of miR-196a-2 and miR-499 variants with ulcerative colitis and their correlation with expression of respective miRNAs," PLoS One, vol. 12, no. 3, article e0173447, 2017.

[48] O. G. Shaker, N. A. El Boghdady, and A. E. El Sayed, “Association of MiRNA-146a, MiRNA-499, IRAK1 and PADI4 polymorphisms with rheumatoid arthritis in Egyptian population," Cellular Physiology and Biochemistry, vol. 46, no. 6, pp. 2239-2249, 2018.

[49] Q. Hu, B. Li, R. She et al., "Association of polymorphisms of miR-146a rs2910164 locus with clinical features of rheumatoid arthritis," Zhonghua Yi Xue Yi Chuan Xue Za Zhi, vol. 36, no. 5, pp. 505-507, 2019.

[50] J. Zhang, B. Yang, B. Ying et al., "Association of premicroRNAs genetic variants with susceptibility in systemic lupus erythematosus," Molecular Biology Reports, vol. 38, no. 3, pp. 1463-1468, 2011.

[51] T. Oner, G. Yenmis, K. Tombulturk et al., “Association of premiRNA-499 rs3746444 and pre-miRNA-146a rs2910164 polymorphisms and susceptibility to Behcet's disease," Genetic Testing and Molecular Biomarkers, vol. 19, no. 8, pp. 424-430, 2015.

[52] H. K. T. Trinh, D. L. Pham, S. C. Kim, R. Y. Kim, H. S. Park, and S. H. Kim, "Association of the miR-196a2, miR-146a, and miR-499 polymorphisms with asthma phenotypes in a Korean population," Molecular Diagnosis \& Therapy, vol. 21, no. 5, pp. 547-554, 2017.

[53] M. Okubo, T. Tahara, T. Shibata et al., "Association study of common genetic variants in pre-microRNAs in patients with ulcerative colitis," Journal of Clinical Immunology, vol. 31, no. 1, pp. 69-73, 2011.

[54] M. Gazouli, I. Papaconstantinou, K. Stamatis et al., "Association study of genetic variants in miRNAs in patients with 
inflammatory bowel disease: preliminary results," Digestive Diseases and Sciences, vol. 58, no. 8, pp. 2324-2328, 2013.

[55] S. Zare-Karizi, Z. K. Vajargah, and R. Mirfakhraie, “Association study of miR-146a rs2910164 and rs57095329 polymorphisms with risk of Behcet's disease," The Journal of Urmia University of Medical Sciences, vol. 29, pp. 530-538, 2018.

[56] B. Yang, J. L. Zhang, Y. Y. Shi et al., “Association study of single nucleotide polymorphisms in pre-miRNA and rheumatoid arthritis in a Han Chinese population," Molecular Biology Reports, vol. 38, no. 8, pp. 4913-4919, 2011.

[57] Y. Inoue, M. Watanabe, N. Inoue et al., "Associations of single nucleotide polymorphisms in precursor-microRNA (miR)-125a and the expression of mature miR-125a with the development and prognosis of autoimmune thyroid diseases," Clinical and Experimental Immunology, vol. 178, no. 2, pp. 229-235, 2014.

[58] L. Wang, H. Zhang, and N. L. Hou, "Correlations of miR146a and IRAK1 gene polymorphisms with ankylosing spondylitis," European Review for Medical and Pharmacological Sciences, vol. 24, no. 11, pp. 6262-6269, 2020.

[59] E. Ridolfi, C. Fenoglio, C. Cantoni et al., "Expression and genetic analysis of MicroRNAs involved in multiple sclerosis," International Journal of Molecular Sciences, vol. 14, no. 3, pp. 4375-4384, 2013.

[60] H. Yu, Y. Liu, L. Zhang et al., "FoxO1 gene confers genetic predisposition to acute anterior uveitis with ankylosing spondylitis," Investigative Ophthalmology \& Visual Science, vol. 55, no. 12, pp. 7970-7974, 2014.

[61] I. Alemán-Ávila, M. Jiménez-Morales, O. Beltrán-Ramírez et al., "Functional polymorphisms inpre-miR146aandpremiR499are associated with systemic lupus erythematosus but not with rheumatoid arthritis or Graves' disease in Mexican patients," Oncotarget, vol. 8, no. 54, pp. 91876-91886, 2017.

[62] X. Luo, W. Yang, D. Q. Ye et al., "A functional variant in microRNA-146a promoter modulates its expression and confers disease risk for systemic lupus erythematosus," PLoS Genetics, vol. 7, no. 6, article e1002128, 2011.

[63] D. Hu, Z. Zhang, X. Ke, H. Kang, and S. Hong, "A functional variant of miRNA-149 confers risk for allergic rhinitis and comorbid asthma in Chinese children," International Journal of Immunogenetics, vol. 44, no. 2, pp. 62-70, 2017.

[64] J. Qi, S. Hou, Q. Zhang et al., "A functional variant of premiRNA-196a2 confers risk for Behcet's disease but not for Vogt-Koyanagi-Harada syndrome or AAU in ankylosing spondylitis," Human Genetics, vol. 132, no. 12, pp. 13951404, 2013.

[65] R. X. Leng, W. Wang, H. Cen et al., "Gene-gene and gene-sex epistatic interactions of MiR146a, IRF5, IKZF1, ETS1 and IL21 in systemic lupus erythematosus," PLoS One, vol. 7, no. 12, article e51090, 2012.

[66] Y. Li, C. Du, W. Wang et al., "Genetic association of MiR146a with multiple sclerosis susceptibility in the Chinese population," Cellular Physiology and Biochemistry, vol. 35, no. 1, pp. 281-291, 2015.

[67] S. E. Löfgren, J. Frostegård, L. Truedsson et al., "Genetic association of miRNA-146a with systemic lupus erythematosus in Europeans through decreased expression of the gene," Genes and Immunity, vol. 13, no. 3, pp. 268-274, 2012.

[68] F. Ghobadi, A. Vaisi-Raygani, F. Bahrehmand et al., "Genetic variants of pre-microRNAs A-499G(rs3746444) and T-196a2C(rs11614913) with ulcerative colitis (UC) and investigated with thiopurine-S-methyltransferase (TPMT) activity," Clinical Laboratory, vol. 63, no. 10/2017, pp. 16831690, 2017.

[69] Z. Golshani, Z. Hojati, A. Sharifzadeh, V. Shaygannejad, and M. Jafarinia, "Genetic variation in intergenic and exonic miRNA sequence and risk of multiple sclerosis in the Isfahan patients," Iranian Journal of Allergy, Asthma, and Immunology, vol. 17, no. 5, pp. 477-484, 2019.

[70] B. Yang, W. Wei, Y. Shi et al., "Genetic variation in miR-146a is not associated with susceptibility to IgA nephropathy in adults from a Chinese Han population," PLoS One, vol. 10, no. 10, article e0139554, 2015.

[71] M. Vreca, M. Andjelkovic, N. Tosic et al., "Impact of alterations in X-linked_IRAK1_ gene and_miR-146a_on susceptibility and clinical manifestations in patients with systemic sclerosis," Immunology Letters, vol. 204, pp. 1-8, 2018.

[72] H. Zhang, J. Pu, X. Wang et al., "IRAK1 rs3027898 C/A polymorphism is associated with risk of rheumatoid arthritis," Rheumatology International, vol. 33, no. 2, pp. 369-375, 2013.

[73] X. Zhou, J. Zhu, H. Zhang, G. Zhou, Y. Huang, and R. Liu, "Is the microRNA-146a (rs2910164) polymorphism associated with rheumatoid arthritis? Association of microRNA-146a (rs2910164) polymorphism and rheumatoid arthritis could depend on gender," Joint, Bone, Spine, vol. 82, no. 3, pp. 166-171, 2015.

[74] Q. Zhou, A. Kijlstra, S. Hou et al., "Lack of association of miR-146a and Ets-1 gene polymorphisms with Fuchs uveitis syndrome in Chinese Han patients," Molecular Vision, vol. 18, pp. 426-430, 2012.

[75] H. B. Hassine, A. Boumiza, R. Sghiri et al., "Micro RNA-146a but not IRAK1 is associated with rheumatoid arthritis in the Tunisian population," Genetic Testing and Molecular Biomarkers, vol. 21, no. 2, pp. 92-96, 2017.

[76] L. Wei, Q. Zhou, S. Hou et al., "MicroRNA-146a and Ets-1 gene polymorphisms are associated with pediatric uveitis," PLoS One, vol. 9, no. 3, article e91199, 2014.

[77] Q. Zhou, S. Hou, L. Liang et al., "MicroRNA-146a and Ets-1 gene polymorphisms in ocular Behcet's disease and VogtKoyanagi-Harada syndrome," Annals of the Rheumatic Diseases, vol. 73, no. 1, pp. 170-176, 2014.

[78] A. Srivastava, P. Nikamo, W. Lohcharoenkal et al., "MicroRNA-146a suppresses IL-17-mediated skin inflammation and is genetically associated with psoriasis," The Journal of Allergy and Clinical Immunology, vol. 139, no. 2, pp. 550$561,2017$.

[79] A. A. Ibrahim, A. Ramadan, A. A. Wahby, M. Hassan, H. M. Soliman, and T. A. Abdel Hamid, "Micro-RNA 196a2 expression and miR-196a2 (rs11614913) polymorphism in T1DM: a pilot study," Journal of Pediatric Endocrinology \& Metabolism, vol. 32, no. 10, pp. 1171-1179, 2019.

[80] A. B. Maharaj, P. Naidoo, T. Ghazi et al., "MiR-146a G/C rs2910164 variation in South African Indian and Caucasian patients with psoriatic arthritis," BMC Medical Genetics, vol. 19, no. 1, p. 48, 2018.

[81] A. Sakoguchi, M. Jinnin, T. Makino et al., "The miR-146a rs2910164 C/G polymorphism is associated with telangiectasia in systemic sclerosis," Clinical and Experimental Dermatology, vol. 38, no. 1, pp. 99-100, 2013. 
[82] Y. Okada, M. Jinnin, T. Makino et al., "MIRSNP rs2910164 of miR-146a is associated with the muscle involvement in polymyositis/dermatomyositis," International Journal of Dermatology, vol. 53, no. 3, pp. 300-304, 2014.

[83] M. H. Hussein, E. A. Toraih, N. M. Aly, E. Riad, and M. S. Fawzy, "A passenger strand variant in miR-196a2 contributes to asthma severity in children and adolescents: a preliminary study," Biochemistry and Cell Biology, vol. 94, no. 4, pp. 347357, 2016.

[84] A. Chatzikyriakidou, P. V. Voulgari, I. Georgiou, and A. A. Drosos, "A polymorphism in the $3^{\prime}$-UTR of interleukin-1 receptor-associated kinase (IRAK1), a target gene of miR146a, is associated with rheumatoid arthritis susceptibility," Joint, Bone, Spine, vol. 77, no. 5, pp. 411-413, 2010.

[85] T. S. Assmann, G. C. K. Duarte, L. A. Brondani et al., "Polymorphisms in genes encoding miR-155 and miR-146a are associated with protection to type 1 diabetes mellitus," Acta Diabetologica, vol. 54, no. 5, pp. 433-441, 2017.

[86] C. Ciccacci, C. Politi, L. Biancone et al., "Polymorphisms in MIR122, MIR196A2, and MIR124A genes are associated with clinical phenotypes in inflammatory bowel diseases," Molecular Diagnosis \& Therapy, vol. 21, no. 1, pp. 107-114, 2017.

[87] T. Cai, J. Li, X. An et al., "Polymorphisms in MIR499A and MIR125A gene are associated with autoimmune thyroid diseases," Molecular and Cellular Endocrinology, vol. 440, pp. 106-115, 2017.

[88] C. Ciccacci, P. Conigliaro, C. Perricone et al., "Polymorphisms in STAT-4, IL-10, PSORS1C1, PTPN2 and MIR146A genes are associated differently with prognostic factors in Italian patients affected by rheumatoid arthritis," Clinical and Experimental Immunology, vol. 186, no. 2, pp. 157-163, 2016.

[89] K. Ahmadi, A. Soleimani, S. Soleimani Motlagh, S. Baharvand Ahmadi, M. Almasian, and A. A. Kiani, "Polymorphisms of pre-miR-499 rs3746444 T/C and pre-miR-146a rs2910164 $\mathrm{C} / \mathrm{G}$ in the autoimmune diseases of rheumatoid arthritis and systemic lupus erythematosus in the west of Iran," Iranian Journal of Public Health, vol. 49, no. 4, pp. 782-790, 2020.

[90] G. Ayeldeen, Y. Nassar, H. Ahmed, O. Shaker, and T. Gheita, "Possible use of miRNAs-146a and -499 expression and their polymorphisms as diagnostic markers for rheumatoid arthritis," Molecular and Cellular Biochemistry, vol. 449, no. 1-2, pp. 145-156, 2018.

[91] E. A. Toraih, N. M. Ismail, A. A. Toraih, M. H. Hussein, and M. S. Fawzy, "Precursor miR-499a variant but not miR-196a2 is associated with rheumatoid arthritis susceptibility in an Egyptian population," Molecular Diagnosis \& Therapy, vol. 20, no. 3, pp. 279-295, 2016.

[92] H. Yu, Y. Liu, L. Bai, A. Kijlstra, and P. Yang, "Predisposition to Behçet's disease and VKH syndrome by genetic variants of miR-182," Journal of Molecular Medicine (Berlin, Germany), vol. 92, no. 9, pp. 961-967, 2014.

[93] S. A. Fattah, M. H. Ghattas, S. M. Saleh, and D. M. AboElmatty, "Pre-micro RNA-499 gene polymorphism rs3746444 T/C is associated with susceptibility to rheumatoid arthritis in Egyptian population," Indian Journal of Clinical Biochemistry, vol. 33, no. 1, pp. 96-101, 2018.

[94] M. Ahmed Ali, O. Gamil Shaker, H. Mohamed Eid, E. Elsayed Mahmoud, E. Mahmoud Ezzat, and S. Nady Gaber, "Relationship between miR-155 and miR-146a polymorphisms and susceptibility to multiple sclerosis in an
Egyptian cohort," Biomed Rep, vol. 12, no. 5, pp. 276-284, 2020.

[95] V. Caputo, C. Strafella, A. Termine et al., "RNAseq-based prioritization revealed COL6A5, COL8A1, COL10A1 and MIR146A as common and differential susceptibility biomarkers for psoriasis and psoriatic arthritis: confirmation from genotyping analysis of 1417 Italian subjects," International Journal of Molecular Sciences, vol. 21, no. 8, p. 2740, 2020.

[96] A. Chatzikyriakidou, P. V. Voulgari, I. Georgiou, and A. A. Drosos, "The role of microRNA-146a (miR-146a) and its target IL-1R-associated kinase (IRAK1) in psoriatic arthritis susceptibility," Scandinavian Journal of Immunology, vol. 71, no. 5, pp. 382-385, 2010.

[97] K. Bogunia-Kubik, B. Wysoczanska, D. Piatek, M. Iwaszko, M. Ciechomska, and J. Swierkot, "Significance of polymorphism and expression of miR-146a and NFkB1 genetic variants in patients with rheumatoid arthritis," Archivum Immunologiae et Therapiae Experimentalis (Warsz), vol. 64, Supplement 1, pp. 131-136, 2016.

[98] M. Xiao, Y. Ma, X. Chen, and B. Kuang, "Single nucleotide polymorphism of miR-149 and susceptibility of rheumatoid arthritis," Zhong Nan Da Xue Xue Bao. Yi Xue Ban, vol. 40, no. 5, pp. 495-498, 2015.

[99] P. P. Zhou, Y. Li, Z. D. Ma, Z. Y. Li, F. Y. Chen, and Y. X. Jiang, "Single nucleotide polymorphisms in the promoter region of mir-133a-1 and in pre-mir-152 rs1707 may contribute to the risk of asthma in a Chinese Han population," European Review for Medical and Pharmacological Sciences, vol. 20, no. 12, pp. 2642-2649, 2016.

[100] G. Kaidonis, M. C. Gillies, S. Abhary et al., "A singlenucleotide polymorphism in the MicroRNA-146a gene is associated with diabetic nephropathy and sight-threatening diabetic retinopathy in Caucasian patients," Acta Diabetologica, vol. 53, no. 4, pp. 643-650, 2016.

[101] E. A. Toraih, M. H. Hussein, E. al Ageeli et al., "Structure and functional impact of seed region variant in MIR-499 gene family in bronchial asthma," Respiratory Research, vol. 18, no. 1, 2017.

[102] I. S. Kiselev, O. G. Kulakova, N. M. Baulina et al., "Variability of the MIR196A2 gene as a risk factor in primary-progressive multiple sclerosis development," Molekuliarnaia Biologiia (Mosk), vol. 53, no. 2, pp. 249-255, 2019.

[103] L. Zhang, J. Wang, D. Che et al., "The association between the miR-146a rs2910164 C>G polymorphism and Kawasaki disease in a southern Chinese population," Bioscience Reports, vol. 38, no. 4, 2018.

[104] W. Ibrahim, B. R. Sakr, E. Obaya, and H. Ghonem, "MicroRNA-146a expression and microRNA-146a rs2910164 polymorphism in Behcet's disease patients," Clinical Rheumatology, vol. 38, no. 2, pp. 397-402, 2019.

[105] M. E. Fouda, "Genetic variants of microRNA-146a gene: an indicator of systemic lupus erythematosus susceptibility, lupus nephritis, and disease activity," Molecular Biology Reports, vol. 47, no. 10, pp. 7459-7466, 2020.

[106] E. T. Massignam, C. Dieter, F. M. Pellenz, T. S. Assmann, and D. Crispim, "Involvement ofmiR-126rs4636297 andmiR146ars2910164 polymorphisms in the susceptibility for diabetic retinopathy: a case-control study in a type 1 diabetes population," Acta Ophthalmologica, vol. 99, no. 4, 2021.

[107] E. Keewan and S. A. Naser, "MiR-146a rs2910164 G > C polymorphism modulates Notch-1/IL-6 signaling during 
infection: a possible risk factor for Crohn's disease," Gut Pathogens, vol. 12, no. 1, p. 48, 2020.

[108] C. Li, W. Fu, Y. Zhang et al., "Meta-analysis of microRNA146a rs2910164 G>C polymorphism association with autoimmune diseases susceptibility, an update based on 24 studies," PLoS One, vol. 10, no. 4, article e0121918, 2015.

[109] R. Duan, C. Pak, and P. Jin, "Single nucleotide polymorphism associated with mature miR-125a alters the processing of primiRNA," Human Molecular Genetics, vol. 16, no. 9, pp. 1124-1131, 2007.

[110] C. Labbaye and U. Testa, "The emerging role of MIR-146A in the control of hematopoiesis, immune function and cancer," Journal of Hematology \& Oncology, vol. 5, no. 1, p. 13, 2012.

[111] K. Jazdzewski, S. Liyanarachchi, M. Swierniak et al., "Polymorphic mature microRNAs from passenger strand of premiR-146a contribute to thyroid cancer," Proceedings of the National Academy of Sciences of the United States of America, vol. 106, no. 5, pp. 1502-1505, 2009.

[112] R. Park, W. J. Lee, and J. D. Ji, “Association between the three functional miR-146a single-nucleotide polymorphisms, rs2910164, rs57095329, and rs2431697, and autoimmune disease susceptibility: a meta-analysis," Autoimmunity, vol. 49, no. 7, pp. 451-458, 2016.

[113] L. Fu, L. Jin, L. Yan et al., "Comprehensive review of genetic association studies and meta-analysis on miRNA polymorphisms and rheumatoid arthritis and systemic lupus erythematosus susceptibility," Human Immunology, vol. 77, no. 1, pp. 1-6, 2016.

[114] The International Consortium for Systemic Lupus Erythematosus Genetics (SLEGEN), J. B. Harley, M. E. AlarcónRiquelme et al., "Genome-wide association scan in women with systemic lupus erythematosus identifies susceptibility variants in_ITGAM_,_PXK_,_KIAA1542_and other loci," Nature Genetics, vol. 40, no. 2, pp. 204-210, 2008.

[115] W. Yang, N. Shen, D. Q. Ye et al., "Genome-wide association study in Asian populations identifies variants in ETS1 and WDFY4 associated with systemic lupus erythematosus," PLoS Genetics, vol. 6, no. 2, article e1000841, 2010.

[116] A. E. Hoffman, T. Zheng, C. Yi et al., "microRNA miR-196a-2 and breast cancer: a genetic and epigenetic association study and functional analysis," Cancer Research, vol. 69, no. 14, pp. 5970-5977, 2009.

[117] M. K. Sibin, S. M. Harshitha, K. V. Narasingarao, I. B. Dhananjaya, P. S. Dhaval, and G. K. Chetan, "Effect of rs11614913 polymorphism on mature miR196a2 expression and its target gene HOXC8 expression in human glioma," Journal of Molecular Neuroscience, vol. 61, no. 2, pp. 144151, 2017.

[118] G. G. Song, S. C. Bae, Y. H. Seo et al., "The association between susceptibility to inflammatory arthritis and miR146a, miR-499 and IRAK1 polymorphisms," Zeitschrift für Rheumatologie, vol. 74, no. 7, pp. 637-645, 2015.

[119] B. Ying, Y. Shi, X. Pan et al., "Association of polymorphisms in the human IL-10 and IL-18 genes with rheumatoid arthritis," Molecular Biology Reports, vol. 38, no. 1, pp. 379-385, 2011.

[120] R. J. Lin and Y. C. Lin, "miR-149* induces apoptosis by inhibiting Akt1 and E2F1 in human cancer cells," Molecular Carcinogenesis, vol. 49, no. 8, pp. 719-727, 2010.
[121] R. S. Redis, S. Calin, Y. Yang, M. J. You, and G. A. Calin, "Cell-to-cell miRNA transfer: from body homeostasis to therapy," Pharmacology \& Therapeutics, vol. 136, no. 2, pp. 169-174, 2012.

[122] S. Santonocito, A. Polizzi, G. Palazzo, and G. Isola, "The emerging role of microRNA in periodontitis: pathophysiology, clinical potential and future molecular perspectives," International Journal of Molecular Sciences, vol. 22, no. 11, p. $5456,2021$. 